\title{
Soil-Corrosion Studies, 1946: Ferrous Metals and Alloys
}

\author{
By Irving A. Denison and Melvin Romanoff
}

\begin{abstract}
This report contains the results of measurements of corrosion made on a variety of wrought and cast ferrous materials after exposure to different soil conditions for periods up to 14 years. The steels ranged in composition from fractional percentages of nickel and chromium to the high concentrations typical of wholly austenitic steels. The soils ranged from well aerated soils deficient in soluble salts to poorly aerated soils containing high concentrations of water-soluble materials. The magnitude and progress of corrosion with respect to both weight loss and pitting are interpreted in relation to the properties of the soils at the test sites. A comparison is made of the corrosion of specimens of plain wrought materials and cast iron.
\end{abstract}

\section{Introduction}

In 1932 the Underground Corrosion Section of the National Bureau of Standards undertook the investigation of the behavior of a variety of ferrous and nonferrous metals and alloys in soils representing a wide range of environmental conditions. The primary objective of the investigation was to ascertain whether metals and alloys that were especially resistant to corrosion in the atmosphere and in natural waters were also resistant to underground corrosion, at least in specific soil environments. It was anticipated that in the course of the investigation the specific effects on corrosion resistance of various alloying elements under different soil conditions might also be evaluated.

Originally, a sufficient number of specimens were buried at 14 test sites to provide for removal of two specimens of each material after each of five periods of exposure. Accordingly, specimens were removed from each test site in 1934, 1937, 1939, 1941, and 1946 and returned to the laboratory for measurement of weight loss and maximum depth of pits. At the time of removal of the specimens in 1937, 1939, and 1941, additional sets of specimens of some of these classes of materials were buried at the test sites and were removed in 1946. Results of exposures of 14, 9, 7 , or $5 \mathrm{yr}$ are, therefore, available for these materials. Corrosion data for the removals prior to 1946 have been published in a series of reports that were summarized by K. H. Logan [1]. ${ }^{1}$

This paper deals only with the tests of wrought and cast ferrous materials. The results of the tests of nonferrous materials will be presented in a subsequent paper.

\section{Properties of the Soils at the Test Sites}

The test sites were selected to represent a wide range of soil conditions, from slightly corrosive to highly corrosive. The considerable differences in the physical and chemical properties of the soils at the test sites are evident from the data of table 1 . The resistivity ranges from $62 \mathrm{ohm}-\mathrm{cm}$, corresponding to a high concentration of soluble salts, to $17,800 \mathrm{ohm}-\mathrm{cm}$, indicating practically no soluble material. The water-soluble material in soil 64, Docas clay, consists of sodium and potassium chlorides, whereas the soluble material in soil 60 , Rifle peat, is composed entirely of sulfates of the alkalies and of the alkaline earth metals. The hydrogen-ion concentration of the soils ranges from $\mathrm{pH} 2.6$ to $\mathrm{pH}$ 9.4. Measurements of $\mathrm{pH}$ and total acidity were made in the laboratory on samples of soil shipped from the test sites in sealed containers. This precaution was taken because results of a previous study showed that aeration of certain poorly aerated soils produced marked increases in acidity because of oxidation of sulfides to sulfates [2].

1 Figures in brackets indicate the literature references at the end of this paper. 
TABLe 1. Properties of the soils at the test sites

\begin{tabular}{|c|c|c|c|c|c|c|c|c|c|c|c|c|c|c|c|c|}
\hline \multicolumn{2}{|r|}{ Soil } & \multirow[b]{2}{*}{ Location } & \multirow[b]{2}{*}{$\begin{array}{l}\text { Aera- } \\
\text { tion a }\end{array}$} & \multirow{2}{*}{$\begin{array}{l}\text { Mois- } \\
\text { ture } \\
\text { equiv- } \\
\text { alent }\end{array}$} & \multirow{2}{*}{$\begin{array}{l}\text { Appar- } \\
\text { ent } \\
\text { specific } \\
\text { gravity }\end{array}$} & \multirow{2}{*}{$\begin{array}{c}\text { Resistiv- } \\
\text { ity at } \\
60^{\circ} \mathrm{F} \\
\left(15.6^{\circ} \mathrm{C}\right)\end{array}$} & \multirow[b]{2}{*}{$\mathrm{pH}$} & \multirow{2}{*}{$\begin{array}{c}\text { Total } \\
\text { acidity b } \\
\text { (milli- } \\
\text { gram } \\
\text { equiv- } \\
\text { alents } \\
\text { per 100 g } \\
\text { of soil) }\end{array}$} & \multirow[b]{2}{*}{$\begin{array}{l}\text { Sulfide } \\
\text { con- } \\
\text { tent } \text { c }\end{array}$} & \multicolumn{7}{|c|}{$\begin{array}{c}\text { Composition of water extract-milligram equivalents per } \\
100 \mathrm{~g} \text { of soil }\end{array}$} \\
\hline No. & Type & & & & & & & & & $\begin{array}{l}\mathrm{Na}+\mathrm{K} \\
\text { as Na }\end{array}$ & $\mathrm{Ca}$ & $\mathrm{Mg}$ & $\mathrm{CO}_{3}$ & $\mathrm{HCO}_{3}$ & $\mathrm{Cl}$ & $\mathrm{SO}_{4}$ \\
\hline 51 & Acadia clay ....... & Spindletop, Tex & $\mathrm{P}$ & $\begin{array}{c}\text { Percent } \\
\quad 47.1\end{array}$ & 2. 07 & $\begin{array}{r}\text { Ohm-cm } \\
190\end{array}$ & 6.2 & 13. 2 & + & 10. 27 & 15.55 & 5. 03 & 0.00 & 0.56 & 5.75 & 22.00 \\
\hline 53 & Cecil clay loam... & Atlanta, Ga....... & G & 33.7 & 1. 60 & 17,800 & 4. 8 & 5.1 & - & & & & & & & \\
\hline 55 & Hagerstown loam... & Loch Raven, Md... & $\mathrm{G}$ & 32.0 & 1.49 & 5,210 & 5.8 & 10.9 & - & & & & & & & \\
\hline 56 & Lake Charles clay & El Vista, Tex & VP & 28.7 & 2. 03 & 406 & 7.1 & 5.1 & + & 3.12 & 0.69 & 0.47 & 0.00 & 0.80 & 1. 59 & 3. 01 \\
\hline 58 & Muck & New Orleans, La ... & $\mathrm{P}$ & 57.8 & 1.43 & 712 & 4. 8 & 15.0 & + & 2.03 & 2. 23 & 1. 29 & .00 & .00 & .47 & 2. 54 \\
\hline 59 & Carlisle muck & Kalamazoo, Mich & VP & 43.6 & & 1,660 & 5.6 & 12.6 & +++ & 1.03 & 3.08 & 2. 70 & .00 & .00 & 3.47 & 1.04 \\
\hline 60 & Rifle peat $\ldots . .$. & Plymouth, Ohio _... & $\mathrm{P}$ & 43.4 & 1. 28 & 218 & 2.6 & 297.4 & ++ & 2. 91 & 10.95 & 2. 86 & .00 & .00 & .00 & 56.70 \\
\hline 61 & Sharkey clay .. & New Orleans, La & $\mathrm{P}$ & 30.8 & 1.78 & 943 & 6.8 & 4.9 & + & 0.73 & 0.68 & 0.33 & .00 & .71 & .10 & 0.91 \\
\hline 62 & Susquehanna clay ... & Meridian, Miss. . . . & $\mathrm{P}$ & 34.6 & 1. 79 & 6,920 & 4.5 & 12.0 & - & & & & & & & \\
\hline 63 & Tidal marsh & Charleston, S. C ..... & $\mathrm{VP}$ & 46. 7 & 1.47 & 84 & 6. 9 & 14.6 & +++ & 33.60 & 6.85 & 4.00 & 0.00 & 0.00 & 12. 70 & 36.60 \\
\hline 64 & Docas clay $\ldots \ldots \ldots$ & Cholame, Calif_..... & $\mathrm{F}$ & 41.1 & 1.88 & 62 & 7.5 & A & - & 28.10 & 2. 29 & .76 & .00 & .89 & 28.80 & .26 \\
\hline 65 & Chino silt loam _. & Wilmington, Calif. & G & 26.4 & 1.41 & 148 & 8.0 & A & - & 7. 65 & 12. 40 & 2. 20 & .00 & 1.30 & 6. 05 & 16. 90 \\
\hline 66 & Mohave fine gravelly loam. & Phcenix, Ariz .... & $\mathrm{F}$ & 16.5 & 1.79 & 232 & 8.0 & A & - & 6.55 & 0.51 & 0.18 & .00 & 0.73 & 2. 77 & 2. 97 \\
\hline 67 & Cinders & Milwaukee, $\mathrm{W}$ is ..... & VP & & & 455 & 7.6 & A & + & 0.77 & 3.03 & .53 & .00 & .55 & 0.09 & 2. 89 \\
\hline 70 & Merced silt loam & Buttonwillow, Calif & $\mathrm{F}$ & 24.7 & 1.69 & 278 & 9.4 & A & - & 8.38 & 0.38 & .22 & .02 & 1.87 & 1.12 & 5. 57 \\
\hline 72 & Papakating silty clay loam & Deerfield, Ohio & VP & & & 762 & 7. 2 & 17 & + & 3.11 & 7. 49 & .95 & & & & 11.58 \\
\hline
\end{tabular}

Aeration of soils: G, good; F, fair; P, poor; VP, very poor.

${ }^{1} \mathrm{~A}$ indicates absence of acidity because of alkaline reaction.

- Sulfides absent; + sulfides present in low concentration; ++ sulfides present in moderate concentration; +++ sulfides present in high concentration. 
The retentiveness of the soils for moisture is indicated by the moisture equivalent, i. e., the quantity of water retained by a previously saturated soil against a centrifugal force of 1,000 times the force of gravity. Values for moisture equivalent range from 16.5 percent for soil 66, Mohave fine gravelly loam, to 57.8 percent for soil 58 , muck.

TABLE 2. Classification of soils according to type of environment

\begin{tabular}{|c|c|c|}
\hline $\begin{array}{l}\text { Soil } \\
\text { No. }\end{array}$ & Soil type & Environment \\
\hline 51 & A cadia clay . . & Inorganic-reducing-acid. \\
\hline 53 & Cecil clay loam ........ & Inorganic-oxidizing-acid. \\
\hline 55 & Hagerstown loam ........... & Do. \\
\hline 56 & Lake Charles clay & Inorganic-reducing-alkaline. \\
\hline 58 & Muck & Organic-reducing-acid. \\
\hline 59 & Carlisle muck . . . . . . . & Do. \\
\hline 60 & Rifle peat ........... & Do. \\
\hline 61 & Sharkey clay ........ & Inorganic-reducing-acid. \\
\hline 62 & Susquehanna clay .......... & Inorganic-oxidizing-acid. \\
\hline 63 & Tidal marsh & Organic-reducing-acid. \\
\hline 64 & Docas clay .......... & Inorganic-reducing-alkaline. \\
\hline 65 & Chino silt loam & Inorganic-oxidizing-alkaline. \\
\hline 66 & Mohave fine gravelly loam. & Do. \\
\hline 67 & Cinders $\ldots \ldots \ldots$ & \\
\hline 70 & Merced silt loam & Inorganic-reducing-alkaline. \\
\hline
\end{tabular}

Values for apparent specific gravity are presented as an index of the relative porosity of the soils. Because the real specific gravities of the mineral portion of soils lie within a narrow range, the apparent specific gravity indicates the compactness and hence the relative porosity of soils that are primarily inorganic in nature.

On the basis of their properties the soils were classified according to type of environment. This classification is given in table 2 .

\section{Description of the Materials}

The forms, dimensions, and compositions of the specimens are given in tables 3 and 4 . Although the identifying letters shown in the table are the same for a few materials, the dates of burial of these specimens are different. As comparisons will be made only between materials exposed for the same length of time, no confusion is likely to result from use of the same symbol for different samples. Specimens of some of the materials were in the form of pipe, others of plate. The ends of the pipe specimens were closed by caps which excluded moisture, thereby preventing corrosion of the interior. As an extra precaution against possible corrosion, the inner surfaces of the specimens were coated with heavy grease. The exposed area of each specimen was approximately $0.3 \mathrm{ft}^{2}$. All of the materials were free of mill scale except as noted in table 3 . 
TABLE 3. Composition of the wrought metals

\begin{tabular}{|c|c|c|c|c|c|c|c|c|c|c|c|c|c|c|c|c|}
\hline Material & $\begin{array}{l}\text { Iden- } \\
\text { tifica- } \\
\text { tion }\end{array}$ & $\begin{array}{c}\text { Year } \\
\text { buried }\end{array}$ & Form & $\begin{array}{c}\text { Nomi- } \\
\text { nal } \\
\text { width } \\
\text { or di- } \\
\text { ameter }\end{array}$ & Length & $\begin{array}{l}\text { Thick- } \\
\text { ness }\end{array}$ & $\mathrm{C}$ & $\mathrm{Si}$ & Mn & s & $\mathrm{P}$ & $\mathrm{Cr}$ & $\mathrm{Ni}$ & $\mathrm{Cu}$ & Mo & Other elements \\
\hline \multicolumn{17}{|c|}{ WROUGHT IRON } \\
\hline $\begin{array}{l}\text { Hand-puddled } \\
\text { Mechanically puddled. }\end{array}$ & $\begin{array}{l}\text { a } \mathrm{A} \\
\text { a } \mathrm{B}\end{array}$ & $\begin{array}{l}1932 \\
1932\end{array}$ & Pipe & $\begin{array}{l}\text { In. } \\
1.5 \\
1.5\end{array}$ & $\begin{array}{l}\text { In } \\
12 \\
12\end{array}$ & $\begin{array}{l}\text { In. } \\
0.145 \\
.145\end{array}$ & $\begin{array}{l}\text { Percent } \\
0.016 \\
.017\end{array}$ & $\begin{array}{l}\text { Percent } \\
0.10 \\
.125\end{array}$ & $\begin{array}{l}\text { Percent } \\
0.029 \\
.041\end{array}$ & $\begin{array}{c}\text { Percent } \\
0.018 \\
.018\end{array}$ & \begin{tabular}{|l} 
Percent \\
0.160 \\
.106
\end{tabular} & $\begin{array}{c}\text { Percent } \\
\cdots-- \\
-\cdots\end{array}$ & \begin{tabular}{|c|} 
Percent \\
$\cdots--$ \\
$-\cdots$
\end{tabular} & $\begin{array}{c}\text { Percent } \\
-\cdots \\
---\end{array}$ & $\begin{array}{c}\text { Percent } \\
--- \\
---\end{array}$ & $\begin{array}{c}\text { Percent } \\
\text { Oxide+slag, } 2.56 . \\
\text { Oxide+slag, } 2.681 \text {. }\end{array}$ \\
\hline \multicolumn{17}{|c|}{ CARBON STEELS } \\
\hline Low-carbon steel & $\mathrm{N}$ & 1932 & Pipe ........ & 2.3 & 10 & 0.145 & 0.15 & -..- & 0.49 & 0.030 & 0.013 & -..- &.-- & -..- & -..- & \\
\hline \multicolumn{17}{|c|}{ LOW-ALLOY IRONS AND STEELS } \\
\hline Open-hearth steel & A & 1937 & Plate & 2.5 & 12 & 0.188 & c. 033 & 0.002 & 0.029 & 0.017 & 0.006 & 0.049 & 0.034 & 0.052 & & \\
\hline Copper-molybdenum open-hearth iron & MM & 1939 & . do n...... & 2.5 & 12 & .250 & .04 & $-\cdots$ & .16 & .027 & .008 & .04 & .14 & .51 & 0.07 & $\begin{array}{l}\mathrm{O}_{2}, \quad 0.015 ; \mathrm{N}_{2}, .008 ; \\
\quad \mathrm{Sn}, 0.002 .\end{array}$ \\
\hline Do.... & $\mathrm{O}$ & 1937 & ..... do & 2.5 & 12 & .243 & .03 & .003 & .16 & .032 & .007 & .02 & .15 & .45 & .07 & \\
\hline Do .... & $\mathrm{N}$ & 1937 & ...... do ...... & 2.5 & 12 & .250 & .06 & .001 & .098 & .029 & .069 & .02 & .14 & .54 & .13 & \\
\hline Do & $\mathrm{H}$ & 1932 & Pipe ....... & 1.5 & 12 & .145 & .04 & .05 & .32 & .027 & .016 & .... & .... & .52 & .15 & \\
\hline Do . & $\mathrm{H}$ & 1941 & .............. & 1.5 & 14 & .145 & .036 & .005 & .16 & .025 & .008 & .... & .... & .47 & .08 & \\
\hline Copper-nickel steel.... & b J & 1937 & Plate & 2.5 & 12 & .265 & .06 & .047 & .49 & .025 & .095 & .... & .52 & .95 & ...- & 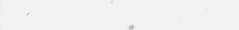 \\
\hline Nickel-copper steel_... & $\mathrm{b} B$ & 1937 & do & 2.5 & 12 & .248 & .07 & .14 & .44 & .022 & .010 & .... & 1.96 & 1.01 & .... & \\
\hline Do & $\mathrm{Y}$ & 1941 & Pipe & 2.0 & 8.5 & .210 & .12 & .12 & .58 & .025 & .07 & ...- & 1.95 & 1.04 & -..- & \\
\hline Do ${ }^{\circ}$ & $\circ \mathrm{P}$ & 1941 & Plate & 2.5 & 14 & .172 & -..- & .... & .... & ...- & .... & -...- & -..- & .... & ...- & \\
\hline Carbon steel layers & $\ldots$ & $\ldots$ & - n & $\ldots$ & $\ldots$ & .036 & .16 & .21 & .78 & .030 & .018 & .... & .... & 0.038 & .... & \\
\hline $\mathrm{Ni}-\mathrm{Cu}$ steel layer. & -..- & ...- & -.......... & .... & -..- & .100 & .14 & .16 & .48 & .009 & .021 & .04 & 1.86 & .98 & .... & \\
\hline Do & $\mathrm{D}$ & 1932 & ..... do & 1.5 & 12 & .145 & .14 & .19 & .21 & .... & .... & .... & 2.47 & 1.08 & .... & \\
\hline $\begin{array}{l}\text { Uhromium-silicon-copper-phosphorus } \\
\text { steel. }\end{array}$ & $\mathrm{C}$ & 1937 & Plate & 2.5 & 12 & .188 & .075 & .84 & .20 & .018 & .124 & 1.02 & .022 & .428 & -..- & \\
\hline $2 \%$ chromium steel with molybdenum & $\mathrm{NN}$ & 1939 & Pipe _....... & 1.5 & 14.5 & .145 & .09 & .25 & .46 & .010 & .015 & 1. 96 & .... & ...- & .49 & \\
\hline Do & $\mathrm{KK}$ & 1937 & Plate & 2.5 & 12 & .175 & .082 & .51 & .46 & .015 & .017 & 2.01 & .07 & .004 & .57 & \\
\hline
\end{tabular}




\begin{tabular}{|c|c|c|c|c|c|c|c|c|c|c|c|c|c|c|c|c|}
\hline \multicolumn{17}{|c|}{ CHROMIUM STEELS } \\
\hline 4 to $6 \%$ chromium steel & $P$ & 1932 & Pipe......... & 2.3 & 10 & 0.154 & 0.13 & .... & 0.46 & 0.025 & 0.012 & 5. 05 & & & .... & \\
\hline Do & $D$ & 1937 & Plate & 2.5 & 12 & .245 & .077 & 0.43 & .37 & .005 & .015 & 5. 02 & 0.09 & 0.008 & .... & \\
\hline 4 to $6 \%$ chromium steel with molyb- & $E$ & 1937 & ..... do & 2.5 & 12 & .188 & .074 & .41 & .32 & .006 & .013 & 4.67 & .09 & .004 & 0.51 & $\mathrm{Al}, 0.030 ; \mathrm{Ti}, 0.022$. \\
\hline $\begin{array}{l}\text { denum. } \\
\text { Do }\end{array}$ & $H$ & 1937 & ..... do _. & 2.5 & 12 & .203 & .060 & .39 & 40 & .014 & .021 & 5.76 & .17 & .004 & 43 & Al 0.27 \\
\hline $12 \%$ chromium steel & $U$ & 1932 & .... do & 4 & 6 & .063 & .065 & .28 & .38 & .017 & .011 & 11.95 & .482 & .0025 & .40 & $A 1,0.26$. \\
\hline $18 \%$ chromium steel & $\mathrm{V}$ & 1932 & do ........ & 4 & 6 & .063 & .070 & .34 & .36 & .015 & .014 & 17.08 & .092 & .021 & $\ldots$ & \\
\hline Do & $x$ & 1932 & Pipe..... & 1.5 & 12 & .145 & .12 & .277 & .42 & .017 & .016 & 17. 72 & .287 &..- & $\cdots$ & \\
\hline \multicolumn{17}{|c|}{ HIGH-CHROMIUM STEELS WITH NICKEL AND MANGANESE } \\
\hline $18 \%$ chromium steel with nickel & $K$ & 1932 & Plate & 3 & 11 & 0.025 & 0.08 & 0.33 & c. 44 & 0.022 & 0.015 & 17. 20 & 8.95 & $\ldots$ & $\ldots$ & \\
\hline Do & $\begin{array}{l}\Lambda \\
R\end{array}$ & $\begin{array}{l}1932 \\
1932\end{array}$ & Pipe..... & 1.5 & 12 & $\begin{array}{r}0.020 \\
.145\end{array}$ & $\begin{array}{r}0.08 \\
.05\end{array}$ & $\begin{array}{r}0.53 \\
.28\end{array}$ & $\begin{array}{r}6.44 \\
.46\end{array}$ & $\begin{array}{r}0.022 \\
.011\end{array}$ & $\begin{array}{r}0.010 \\
.015\end{array}$ & $\begin{array}{l}1.20 \\
17.52\end{array}$ & $\begin{array}{l}8.90 \\
8.85\end{array}$ & $\cdots$ & $\cdots$ & \\
\hline Do & $W$ & 1932 & Plate..... & 4 & 6 & .063 & .093 & .42 & .36 & .017 & .008 & 18. 69 & $\begin{array}{l}0.00 \\
9.18\end{array}$ & 0.016 & $\ldots$ & \\
\hline $\begin{array}{l}18 \% \text { chromium steel with nickel and } \\
\text { manganese. }\end{array}$ & $T$ & 1932 & ..... do & 6 & 10 & .063 & .06 & .40 & 6.09 & -.. & -... & 17. 76 & 3. 83 & .95 & $\ldots$ & \\
\hline Do & $S$ & 1932 & $\ldots$.... do ... & 6 & 10 & .063 & .07 & .48 & 9.44 & .... & .... & 17. 78 & .... & .74 & .... & \\
\hline $18 \%$ chromium steel with nickel, man- & $C M$ & 1939 & - do .... & 2 & 12.5 & .25 & .07 & .40 & 1. 24 & .008 & .016 & 17.78 & 10.96 & $\cdots$ & 2. 63 & \\
\hline $\begin{array}{l}22 \% \text { chromium steel with nickel and } \\
\text { manganese. }\end{array}$ & Y & 1932 & -.... do & 4 & 6 & .063 & .144 & .59 & 1. 80 & .011 & .015 & 22.68 & 12. 94 & .021 & $\cdots$ & \\
\hline $\begin{array}{l}22 \% \text { nickel-chromium steel with man- } \\
\text { ganese and molybdenum. }\end{array}$ & $D T$ & 1939 & $\ldots$..._. do & 2.5 & 12 & .25 & .07 & .91 & 1. 99 & .012 & .014 & 19. 27 & 22.12 & 1.07 & 3. 52 & \\
\hline
\end{tabular}

a Some mill scale on the surface at the time of burial.

b Specimens completely covered with a hard, black mill scale at the time of burial.

- Specimens composed of three layers as follows: outer layers-plain carbon steel, 0.036 in. thick; intermediate layer-2-percent nickel, 1-percent copper steel, 0.100 in. thick. 
TABLE 4. Composition of the cast metals

\begin{tabular}{|c|c|c|c|c|c|c|c|c|c|c|c|c|c|c|c|c|}
\hline \multirow{2}{*}{ Material } & \multirow{2}{*}{$\begin{array}{l}\text { Identi- } \\
\text { fication }\end{array}$} & \multirow{2}{*}{$\begin{array}{l}\text { Year } \\
\text { buried }\end{array}$} & \multirow{2}{*}{ Form } & \multirow{2}{*}{$\begin{array}{l}\text { Nomi- } \\
\text { nal } \\
\text { width } \\
\text { or di- } \\
\text { ameter }\end{array}$} & \multirow{2}{*}{ Length } & \multirow{2}{*}{$\begin{array}{c}\text { Thick- } \\
\text { ness }\end{array}$} & \multicolumn{3}{|c|}{$\mathrm{C}$} & \multirow{2}{*}{$\mathrm{Si}$} & \multirow{2}{*}{$\mathrm{Mn}$} & \multirow{2}{*}{$\mathrm{S}$} & \multirow{2}{*}{$\mathrm{P}$} & \multirow{2}{*}{$\mathrm{Cr}$} & \multirow{2}{*}{$\mathrm{Ni}$} & \multirow{2}{*}{$\mathrm{Cu}$} \\
\hline & & & & & & & Free & $\begin{array}{l}\text { Com- } \\
\text { hined }\end{array}$ & Total & & & & & & & \\
\hline Rattled cast iron ${ }^{a}$ & G & 1932 & Pipe ... & $\begin{array}{l}\text { Inch } \\
1.25\end{array}$ & $\begin{array}{l}\text { Inch } \\
13.5\end{array}$ & $\begin{array}{l}\text { Inch } \\
0.250\end{array}$ & $\begin{array}{l}\text { Per- } \\
\text { cent } \\
2.94\end{array}$ & $\begin{array}{c}\text { Percent } \\
0.64\end{array}$ & $\begin{array}{c}\text { Percent } \\
3.58\end{array}$ & $\begin{array}{l}\text { Per- } \\
\text { cent } \\
1.64\end{array}$ & $\begin{array}{l}\text { Por- } \\
\text { cent } \\
0.48\end{array}$ & $\begin{array}{l}\text { Per- } \\
\text { cent } \\
0.074\end{array}$ & $\begin{array}{l}\text { Per- } \\
\text { cent } \\
0.79\end{array}$ & $\begin{array}{l}\text { Per- } \\
\text { cent }\end{array}$ & $\begin{array}{l}\text { Per- } \\
\text { cent }\end{array}$ & $\begin{array}{l}\text { Per- } \\
\text { cent }\end{array}$ \\
\hline $\begin{array}{l}\text { Sand-coated cast } \\
\text { iron. }\end{array}$ & $\mathrm{F}$ & 1932 & $\ldots$ do & 1.25 & 13.5 & .250 & 2. 94 & .64 & 3.58 & 1. 64 & .48 & .074 & .79 & ... & $\ldots$ & $\ldots$ \\
\hline Plain cast iron & A & 1941 & _._. do & 1.5 & 13 & .250 & -... & - n-..- & 3.22 & 2.19 & .91 & .12 & -...- & -... & -.... & -.... \\
\hline Low-alloy cast iron. & $\mathrm{I}$ & 1932 & _... do ..... & 1. 25 & 12 & .350 & $-\ldots$ & - n & 2. 53 & 1. 43 & .28 & .077 & .128 & .... & ..... & 0.51 \\
\hline Do & $\mathrm{J}$ & 1932 & ... do & 1.25 & 12 & .350 & $-\ldots$ & -...- & 2. 90 & 2. 04 & .83 & .060 & .248 & -... & $\ldots$ & .62 \\
\hline Do _.............. & $\mathrm{C}$ & 1932 & ... do do...... & 1.5 & 12 & .250 & 3.00 & .50 & 3.50 & 2. 50 & .70 & .050 & .400 & 0.30 & 0.15 & -... \\
\hline Do $\ldots \ldots \ldots \ldots$ & $\mathrm{B}$ & 1941 & ... do & 1.5 & 13 & .250 & $\ldots$ & -..... & 3.28 & 2.09 & .83 & .12 & ..... & .... & 1. 27 & .32 \\
\hline Do $\ldots \ldots \ldots$ & $\mathrm{C}$ & 1941 & ... do do..... & 1.5 & 13 & .250 & -... & (n.... & 3.24 & 2. 08 & .80 & .12 & .... & $\ldots$ & 1. 71 & .98 \\
\hline Do ............... & $\mathrm{NC}$ & 1941 & Plate.... & 2.5 & 14 & .5 & $\ldots$ & - n & 2.80 & 2. 03 & -... & - ... & -...- & $\ldots$ & 2. 08 & 1. 10 \\
\hline Do $\ldots \ldots \ldots$ & $\mathrm{N}$ & 1941 & _. $\mathrm{do}_{\ldots} \ldots \ldots$ & 2.5 & 14 & .5 & $\ldots$ & - n & 2.75 & 2.00 & -... & .... & - . - & $\ldots$ & 3.10 & -.... \\
\hline Do $\ldots \ldots$ & $\mathrm{D}$ & 1941 & Pipe..... & 1.5 & 13 & .250 & $\ldots$ & n. & 3.21 & 2.11 & .72 & .12 & -... & .... & 3.32 & -... \\
\hline High-alloy cast iron & $\mathrm{E}$ & 1932 & $\ldots$. do & 1.5 & 10 & .250 & $\ldots$ & - n. & 2. 98 & 2.13 & 1.00 & $\ldots$ & $\ldots$ & 2. 61 & 15.00 & 6.58 \\
\hline
\end{tabular}

a Ordinary iron horizontally cast in green sand molds and rattled to remove sand.

\section{Results of Exposure Tests}

\section{Effect of Composition}

(a) Low-Alloy Wrought Materials

Prior to examination the specimens were cleaned free of corrosion products by methods that have been previously described [1]. The extent of corrosion was measured by the loss in weight after exposure and by the depths of the deepest pits. These data are recorded in tables 5 and 6 , respec- tively. Unless indicated otherwise each value is the average of measurements made on two specimens. Except as noted in tables 5 and 6 , the exposure periods at the different test sites did not differ by more than 5 percent. A typical set of specimens, including ferrous, nonferrous, and miscellaneous materials, is shown in figure 1 . The condition of various wrought and cast ferrous materials after exposure for 14 years in three different environments is shown in figure 2.

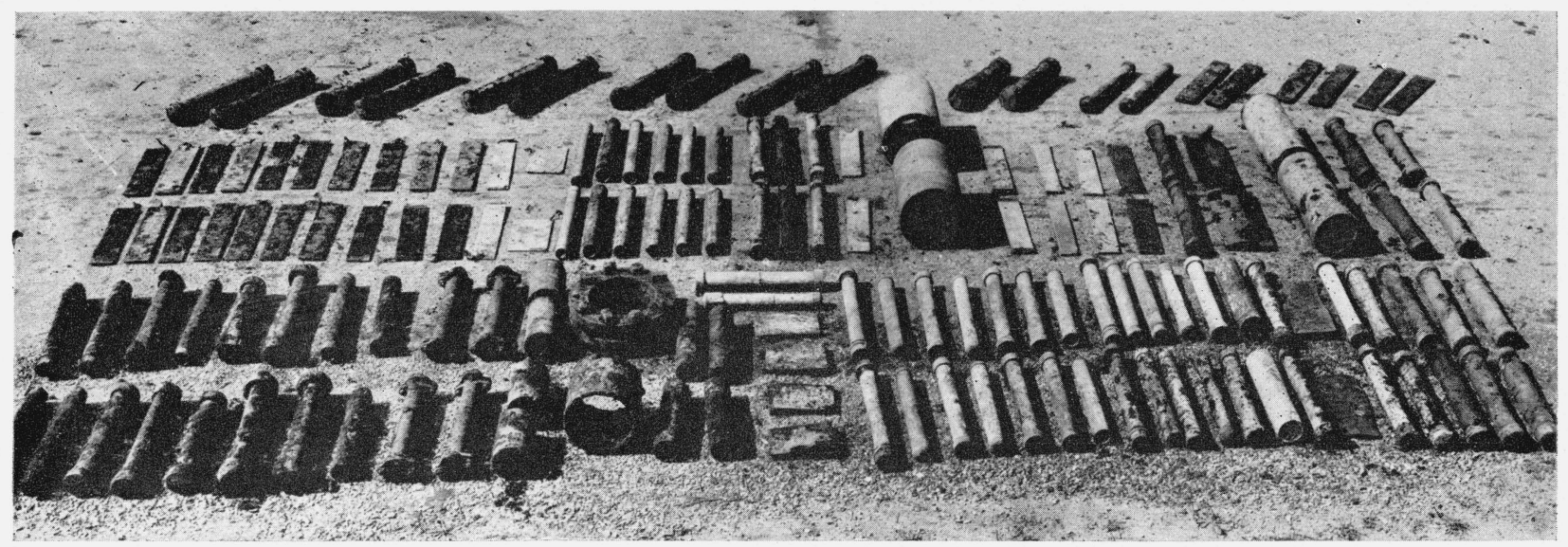

Figure 1. Specimens removed in 1946 from the test site in Hagerstown loam at Loch Raven, Md. 


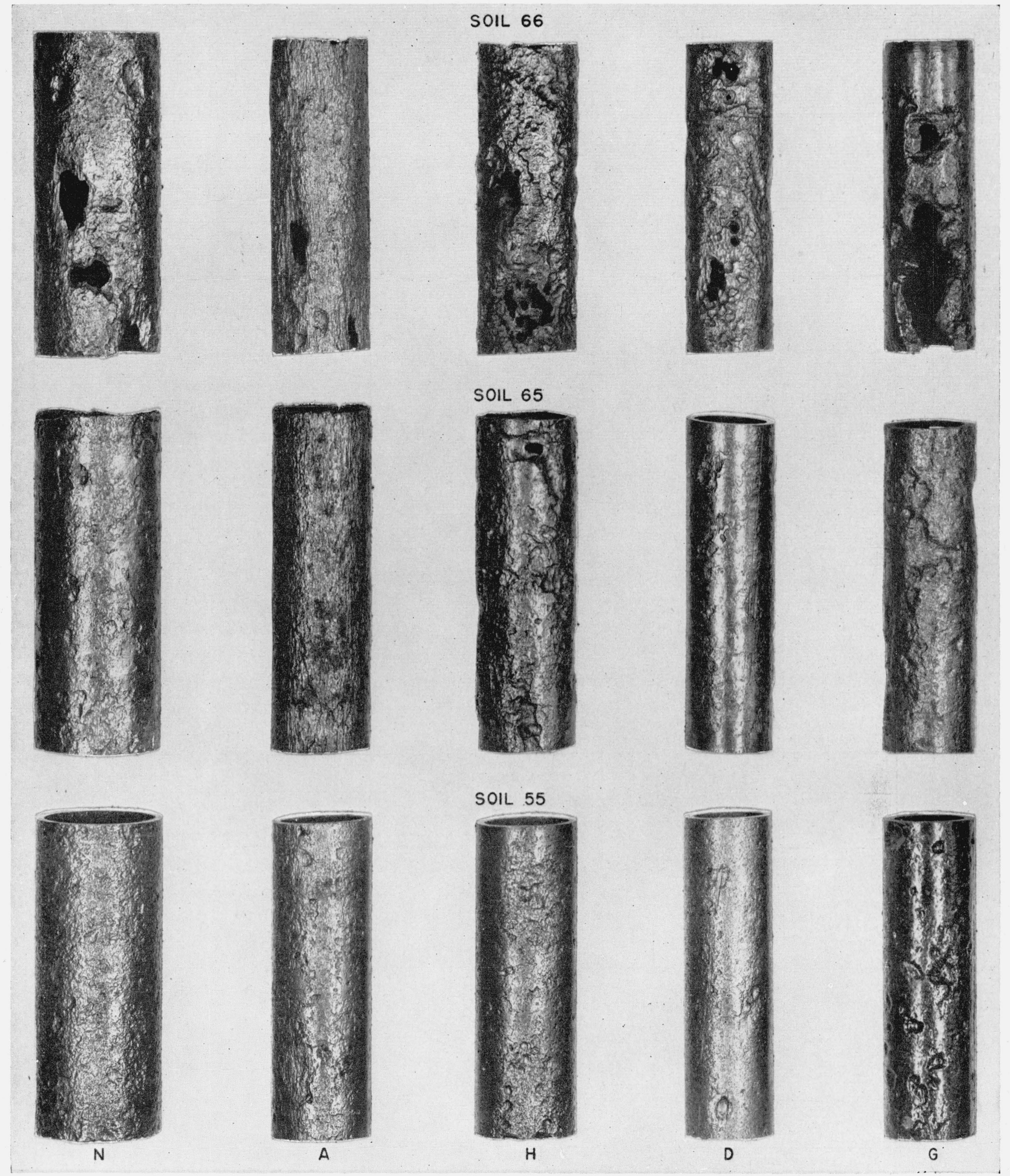

Figure 2. Corrosion of steel, cast iron, and low-alloy iron and steel in soils differing in corrosiveness-exposure 14 yr. N, Carbon steel; A, hand-puddled wrought iron; H, Cu-Mo open-hearth iron; D, 2-percent Ni, 1-percent Cu steel; G. cast iron. 
TABLE 5. Loss in weight of wrought materials

(A verage of two specimens in ounces per square foot)

\begin{tabular}{|c|c|c|c|c|c|c|c|c|c|c|c|c|c|c|c|c|c|c|c|c|}
\hline \multirow[b]{2}{*}{$\begin{array}{l}\text { Identifica- } \\
\text { tion }\end{array}$} & \multirow[b]{2}{*}{$\begin{array}{l}\text { Year } \\
\text { bur- } \\
\text { ied }\end{array}$} & \multirow[b]{2}{*}{ Material } & \multirow[b]{2}{*}{ Form } & \multirow[b]{2}{*}{$\begin{array}{l}\text { A ver- } \\
\text { age } \\
\text { expo- } \\
\text { sure }\end{array}$} & \multicolumn{16}{|c|}{ Test site number and soil type } \\
\hline & & & & & $\begin{array}{c}51 \\
\begin{array}{c}\text { Acadia } \\
\text { clay }\end{array}\end{array}$ & \begin{tabular}{c|}
53 \\
\\
Cecil \\
clay \\
loam
\end{tabular} & $\begin{array}{c}55 \\
\\
\text { Hag- } \\
\text { ers- } \\
\text { town } \\
\text { loam }\end{array}$ & $\begin{array}{c}56 \\
\begin{array}{c}\text { Lake } \\
\text { Charles } \\
\text { clay }\end{array}\end{array}$ & Muck & $\begin{array}{c}59 \\
\\
\text { Car- } \\
\text { lisle } \\
\text { muck }\end{array}$ & $\begin{array}{l}\text { Rifle } \\
\text { peat }\end{array}$ & $\begin{array}{l}61 \\
\\
\text { Shar- } \\
\text { key } \\
\text { clay }\end{array}$ & $\begin{array}{c}62 \\
\\
\text { Susque- } \\
\text { hanna } \\
\text { clay }\end{array}$ & $\begin{array}{l}\text { Tidal } \\
\text { marsh }\end{array}$ & $\begin{array}{c}\text { Docas } \\
\text { clay }\end{array}$ & $\begin{array}{c}65 \\
\begin{array}{c}\text { Chino } \\
\text { silt } \\
\text { loam }\end{array}\end{array}$ & $\begin{array}{c}66 \\
\text { Mo- } \\
\text { have } \\
\text { fine } \\
\text { gravel- } \\
\text { ly loam }\end{array}$ & $\begin{array}{l}\text { Cin- } \\
\text { ders }\end{array}$ & $\begin{array}{c}70 \\
\text { Mer- } \\
\text { ced } \\
\text { silt } \\
\text { loam }\end{array}$ & $\begin{array}{c}72 \\
\text { Papa- } \\
\text { kating } \\
\text { silty } \\
\text { clay } \\
\text { loam }\end{array}$ \\
\hline \multirow{8}{*}{$A_{\ldots}}$. & \multirow{6}{*}{1932} & \multirow{6}{*}{$\begin{array}{l}\text { Wrought iron, hand-pud- } \\
\text { dled. }\end{array}$} & \multirow{8}{*}{ Pipe $\ldots . .-$} & $\begin{array}{r}\text { Years } \\
2.0\end{array}$ & 11.7 & 3.5 & 2.8 & 3.5 & 3.5 & & 5.7 & $A 1.3$ & 3. 0 & 3. 0 & 11.4 & 8.0 & 8.6 & 8.6 & & \\
\hline & & & & 5.4 & 12.6 & 2. 6 & 2.3 & 10.8 & 9.8 & 1.8 & 6.3 & 5.6 & 4. 0 & 3. 1 & 22.1 & 7.4 & 10.2 & 31.8 & & 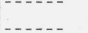 \\
\hline & & & & 7.4 & 15.1 & 3.3 & 3.5 & 17.2 & 11.9 & 2.0 & 5.1 & 6.3 & 6.0 & 3.4 & 34.4 & 9.0 & 11. 6 & 29.7 & & ........ \\
\hline & & & & 9.3 & the & 3.7 & 3.7 & b 22.8 & 12.6 & 2.4 & d 14.3 & 6.4 & 7.8 & d 8.5 & $.16 .0+$ & 13.6 & b 5.8 & $\cdot 15.2+$ & & \\
\hline & & & & 14.3 & e 23.9 & 4. 9 & 3.4 & 26.6 & 19.6 & $\bullet 4.3$ & 25.1 & 10.2 & 8.3 & d 10.1 & $\cdot 38.3+$ & 10.4 & 20.3 & ${ }^{1} \mathrm{D}$ & & ....... \\
\hline & & & & 2.0 & 8.2 & 3.4 & 2. 9 & 4.4 & 3.2 & & 5.0 & \& 1.2 & 4.0 & 2. 6 & 13.3 & 6.2 & 7.8 & 11.4 & & -...... \\
\hline & \multirow{5}{*}{1932} & \multirow{3}{*}{$\begin{array}{l}\text { Wrought iron, mechani- } \\
\text { cally puddled. }\end{array}$} & & 5.4 & 13.6 & 3.0 & 2.4 & 7.6 & 10.4 & 1.6 & 6.8 & 4.9 & 4.0 & 2.4 & 23.1 & 7.2 & 11.3 & 24. 9 & & ....... \\
\hline & & & & 7.4 & 15.3 & 3.4 & 3.4 & 14.7 & 11.6 & 1.8 & 5.4 & 6.4 & 6.0 & 3.5 & 35.4 & 8.8 & 11.1 & 27.0 & & ....... \\
\hline & & & ... do & 9.3 & & 3.7 & 3.8 & 19.5 & 12.7 & 2.3 & d 16.5 & 5.7 & 9.4 & 4.2 & - $18.4+$ & 11.4 & 10.0 & $\mathrm{D}$ & & ....... \\
\hline \multirow{6}{*}{$\mathrm{N}$} & & \multirow[b]{4}{*}{ Low-carbon steel_. } & \multirow{5}{*}{$\ldots$ do $\ldots$} & 14.3 & e 26.7 & 4. 8 & 3.7 & 26.5 & 17.4 & $\cdot 4.2$ & 28.8 & 11.9 & 7.1 & $\mathrm{~d} 6.8$ & $\circ 36.8+$ & 9.2 & - $17.2+$ & $\mathrm{D}$ & & $\ldots$ \\
\hline & & & & 2.0 & 7.4 & 2.7 & 2.4 & 4.0 & 3.2 & & 6. 2 & $=0.8$ & 4.1 & 3.8 & 12.6 & 7.4 & 7.7 & 21.5 & & ........ \\
\hline & \multirow{4}{*}{1932} & & & 5.4 & 12.7 & 3.0 & 2. 2 & 13.9 & 11.2 & 2.4 & 11. 0 & 4.0 & 4.7 & 4. 5 & 25.3 & 10.3 & 15.1 & 34.6 & & 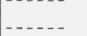 \\
\hline & & & & 7.4 & 11.5 & 4. 2 & 3.2 & 21.0 & 14.1 & 3.0 & 7.6 & 5. 6 & 5.3 & 7.1 & 35.6 & 13.7 & 14.3 & 23.5 & & ....... \\
\hline & & & & 9.3 & & 4. 1 & 38 & 28.8 & 16.2 & 4.7 & 16.7 & 5.8 & 6.6 & d 9.0 & $\mathrm{D}$ & 12.9 & 18.6 & .58.4+ & & ....... \\
\hline & & & & 14.3 & e 21.9 & 4.4 & 3.1 & 35.2 & 25.5 & • 3.9 & 28.8 & 10.0 & 7.9 & 9.6 & D & 13.0 & $\mathrm{D}$ & D & ...... & -.... \\
\hline & & & & 2.1 & 7.5 & 1.8 & 1.8 & 13.8 & 5.1 & 1.5 & 4.0 & 2. 2 & 3.2 & 2.7 & 8.7 & 4.3 & 9.2 & 40.5 & 4. 9 & ....... \\
\hline S $\ldots \ldots$ & 1937 & Carbon steel ... & _._do do... & 4.0 & & 2.9 & 2.6 & 16.0 & 8.8 & 3.3 & 8.1 & 5.0 & 4.3 & 9.2 & 6. 0 & 4.6 & 12.3 & 37.0 & 9.7 & -....- \\
\hline & & & & 9.0 & g 17.3 & 3.4 & 4.1 & 27.8 & 17.3 & 7.5 & 17.6 & 4. 2 & 5.3 & 10.7 & 4.7 & 7. 0 & d 8.0 & 31.6 & 13.4 & ....... \\
\hline & & & & 2 & 11.6 & 1.8 & 2.0 & 14.4 & 5.7 & 1.5 & 6.3 & 2.6 & 2.8 & 3.6 & 7.1 & 4.6 & 8.3 & 12.0 & 5.0 & \\
\hline A $\ldots$ & 1937 & Open-hearth steel.... & Plate $\ldots$ & 4 & & 3. 2 & 2.6 & 18. 4 & 9.9 & 4.2 & 9. 5 & 5.4 & 3.7 & b 6.2 & 7.4 & 5.3 & b 16.8 & 34.3 & 10.6 & $\cdots$ \\
\hline & & & & 9 & g 19.1 & 3.9 & 3.8 & 28.0 & 16.9 & 9.9 & 22.0 & 4.3 & 4.2 & b 8.9 & 7.5 & 7.2 & 4.6 & $\mathrm{D}$ & 17. 9 & ..... \\
\hline & & & & 2.0 & 6. 2 & 2.8 & 2. 0 & 2.3 & 2.9 & & 4. 9 & × 0.9 & 3.7 & 2.5 & 16.1 & 7.5 & 8.8 & 7.8 & & \\
\hline & & & & 5.4 & 11.6 & 2.8 & 1.8 & 8.8 & 10.4 & 1.7 & 5.8 & 4.1 & 4.1 & 3.0 & 23.4 & 11. 2 & 14.4 & 25.0 & & \\
\hline $\mathrm{H}_{\ldots} \ldots$ & 1932 & Open-hearth iron; $0.52 \mathrm{Cu}$, & Pipe .. & 7.4 & 11.6 & 3.9 & 3.4 & 13.0 & 12.2 & 2.6 & 4.5 & 4. 9 & 5.0 & 4.8 & 34.6 & 14.7 & 14. 3 & 13.8 & & \\
\hline & & 0.15 Mo. & & 9.3 & & 4.0 & 3.2 & ${ }_{b} 18.9$ & 14.5 & 2.4 & 13.7 & 5.6 & 7.0 & 6.3 & $\mathrm{D}$ & 15.0 & 14.6 & $\mathrm{D}$ & & $\ldots$ \\
\hline & & & & 14.3 & e 21.9 & 4.2 & 3.2 & 23. 0 & 17.3 & e 4.3 & 25.5 & 9.8 & 5. 9 & $b 8.0$ & 31.5 & 14.9 & $\mathrm{D}$ & $\mathrm{D}$ & ...... & \\
\hline & & & & 2.1 & 7.5 & 2.1 & 2.0 & 12.4 & 5.8 & 1.3 & 4.1 & 3,0 & 3.0 & ${ }_{6} 2.5$ & 8.0 & 5.0 & 9.1 & 33.8 & 5.4 & -... \\
\hline O_.... & 1937 & Open-hearth iron; $0.45 \mathrm{Cu}$, & Plate & 4.0 & & 2.9 & 2.8 & 13.8 & 9.9 & 2.9 & 7.4 & 5.6 & 3.6 & 4.7 & 8.0 & 5. 0 & 11.6 & 33.2 & d 11.0 & ...... \\
\hline & & 0.07 Mo. & & 9.0 & g 19.0 & 3.4 & 4.7 & 28.4 & 17.3 & 7.8 & 16.4 & 4.8 & 4.6 & 7.1 & 6.4 & 6.4 & 5. 9 & 25.1 & 13. 6 & - \\
\hline & & & & 2.1 & 7.2 & 2.0 & 1.9 & 13.0 & 5.5 & 1.4 & 4.8 & 2.7 & 3.0 & 3.0 & 8.0 & 4.6 & 7.4 & 20.9 & 4.8 & \\
\hline $\mathrm{N}_{\ldots} \ldots \ldots$ & 1937 & Open-hearth iron; $0.54 \mathrm{Cu}$, & ... do & 4.0 & & 3.0 & 2.7 & 17.4 & 9.4 & 2. 9 & 8.2 & 5.6 & 3.7 & 4.7 & 6.6 & 5. 0 & 12.0 & 27.7 & d 10.2 & \\
\hline & & 0.13 Mo. & & 9.0 & g 18.8 & 3.3 & 4.6 & 33.7 & 17.0 & 7.6 & 14.5 & 4. 6 & 4.7 & 7.2 & 5.8 & 6.7 & d 6.9 & 21.9 & 15.0 & .... \\
\hline MM & 1939 & Open-hearth iron; $0.51 \mathrm{Cu}$, & ... do & 1.9 & & b 2.3 & 0.9 & 11. 2 & 4.7 & b2.2 & 3.4 & 2.3 & 3.0 & 2.1 & 4.1 & $b 3.1$ & 6.6 & 26.0 & 10.1 & \\
\hline & & 0.07 Mo. & & 6.9 & b 24.1 & 3.6 & 1.8 & 45.8 & 12.7 & 4.6 & 8.4 & 6.4 & 5.2 & 6.7 & b 20.2 & 4. 9 & 12.4 & 27.9 & 11.0 & \\
\hline $\mathrm{H}_{\ldots}$. & 1941 & Open-hearth iron; $0.47 \mathrm{Cu}$, & Pipe _.... & 5.0 & (n-.... & 3.7 & 2.0 & 6.3 & 5.0 & 5.5 & 5.3 & 3.0 & 3.4 & 3.5 & 20.4 & 5.7 & 17.5 & $\mathrm{D}$ & 6.0 & 2.0 \\
\hline & & & & 2.1 & 7.0 & 0.8 & 1.2 & 14.0 & 5.2 & 1.6 & 5.3 & 2.3 & 2.4 & 2.5 & 9.0 & 3.4 & 7.3 & 20.3 & 4.2 & \\
\hline $\mathrm{J}_{\ldots} \ldots$ & 1937 & Copper-nickel steel; 0.95 & Plate ... & 4.0 & 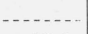 & 1.3 & 1.8 & 19. 2 & 10.6 & 2.6 & 8.6 & 4.8 & 3.3 & 3.5 & 6.0 & 5. 0 & 10.4 & 35.1 & d 7.6 & - \\
\hline & & $\mathrm{Cu}, 0.52 \mathrm{Ni}$ & & 9.0 & g 18.1 & 2.4 & 3.5 & 34.6 & 16.0 & 5.5 & 18.8 & 3.9 & 4.0 & 7.0 & 4.7 & 6. 2 & d 8.2 & 30.4 & 17.3 & - n \\
\hline & & & & 2.1 & 7.4 & 0.6 & 0.8 & 13.0 & 6.2 & 1.4 & 6.4 & 2. 0 & 2.1 & 2. 3 & 6.6 & 4.9 & 8.9 & 23.0 & 3. 9 & ...... \\
\hline B $\ldots \ldots$ & 1937 & Nickel-copper steel; 1.96 & ....do_..... & 4.0 & ........ & 1.1 & 1.2 & 19.5 & 11.0 & 2.4 & 9.6 & 3.9 & 2.9 & 3.4 & 4.9 & 5. 0 & 11.4 & 44.3 & 7.8 & - \\
\hline & & $\mathrm{Ni}, 1.01 \mathrm{Cu}$ & & 9.0 & в 20.2 & 2.8 & 2.6 & 31.2 & 17.7 & 7.2 & 24.1 & 3. 2 & 2. 9 & 8.9 & 7.1 & 7.6 & 3.1 & $\mathrm{D}$ & 18.4 & \\
\hline
\end{tabular}




\begin{tabular}{|c|c|c|c|c|c|c|c|c|c|c|c|c|c|c|c|c|c|c|c|c|}
\hline \multirow{4}{*}{$\mathrm{D} \ldots \ldots$} & \multirow{4}{*}{1932} & \multirow{4}{*}{$\begin{array}{l}\text { Nickel-copper steel; } 2.47 \\
\text { Ni, } 1.08 \mathrm{Cu} \text {. }\end{array}$} & \multirow{4}{*}{ Pipe } & 2. 0 & 4. 7 & 2. 2 & 1. 7 & 3.3 & 3.5 & & 4. 9 & a 1.2 & 2. 7 & 1.6 & 6.5 & 5. 0 & 5.1 & 16.4 & & \\
\hline & & & & 5.4 & 8.3 & 2. 2 & 1.4 & 5.1 & 7.7 & 1. 7 & 7.8 & 2. 6 & 2.3 & 2.3 & 23.6 & 4. 2 & 8.4 & 23.5 & & \\
\hline & & & & 7.4 & 9.6 & 2.6 & 2. 2 & 9.7 & 9.7 & 2. 9 & 3.8 & 3.8 & 3.7 & 4. 1 & 37.6 & 6.1 & 9.2 & 27.5 & & \\
\hline & & & & 9.3 & & 2. 5 & 2. 0 & 16.7 & 6.6 & 2.6 & d 10.3 & 3.4 & 5.4 & 7.6 & b24. 8 & 6. 0 & d 7.5 & $\mathrm{D}$ & & \\
\hline & & & & 14.3 & $\cdot 15.9$ & 3.4 & 2.4 & 14.5 & 13.1 & e 3.7 & 17.4 & 6. 2 & 3.6 & 10.4 & 25.5 & 6.9 & 14.6 & $\mathrm{D}$ & & \\
\hline & 1941 & $\begin{array}{l}\text { Nickel-copper steel; } 1.95 \\
\mathrm{Ni}, 1.04 \mathrm{Cu} \text {. }\end{array}$ & -..do & 5.0 & . & 3.6 & 2. 0 & 0.6 & 3.4 & 4.6 & 3.3 & 2. 6 & 3. 5 & 1. 2 & 20.4 & 3.9 & 15.8 & $\mathrm{D}$ & 6.9 & 2. 1 \\
\hline $\mathrm{P}_{\ldots}$ & 1941 & Composite steel i & Plate $e_{\ldots}$ & 5.0 & & 4.6 & 4. 1 & & 6.2 & 5. 6 & 5.7 & 3.9 & & b 5.9 & 13.0 & 5. 7 & 11.3 & & 6. 2 & 3. 0 \\
\hline & & & & 2.1 & 9.2 & 1.5 & 1.3 & 12.5 & 4.4 & 1.1 & 5.8 & 2.4 & 1.9 & 2.4 & 4.9 & 4. 4 & 6.9 & 17.7 & 5.4 & \\
\hline $\mathrm{C}_{\ldots} .$. & 1937 & Cr-Si-Cu-P steel, $1.02 \mathrm{Cr}$, & ... do do... & 4. 0 & & 2. 4 & 2.3 & 20.8 & 9.0 & 2. 5 & 10.2 & 4.6 & 3.0 & 4.1 & 5.1 & 5.4 & 13.6 & 13.9 & 9.8 & \\
\hline & & $0.42 \mathrm{Cu}$. & & 9.0 & g 19.6 & 3.3 & 3.0 & 28.1 & 16. 7 & 7.8 & 24.9 & 3.6 & 3.8 & b 5.8 & 5.7 & 11.0 & 3.9 & 26.3 & 15.8 & \\
\hline $\mathrm{NN}_{-}$ & 1939 & 2 percent chromium steel & Pipe_ & 1.9 & & 2. 0 & 0.9 & 13.8 & 5.9 & 1.9 & 4.1 & 2. 0 & 2.6 & 1.8 & 3.9 & 3.9 & 9.0 & 17.8 & 12.8 & \\
\hline & & with Mo. & & 6.9 & b 27.1 & b2. 9 & & 46. 0 & 15.6 & 4. 0 & 9.8 & 5. 8 & b 4.4 & 5. 0 & 15.8 & 7. 2 & 18.0 & 23.5 & 12. 1 & \\
\hline KK. & 1937 & $\ldots \mathrm{d}_{\ldots} \ldots \ldots$ & Plate & 4.0 & 1.9 & $\begin{array}{l}1.6 \\
2.2\end{array}$ & $\begin{array}{l}1.6 \\
2.4\end{array}$ & $\begin{array}{r}9.0 \\
14.5\end{array}$ & $\begin{array}{l}3.3 \\
8.4\end{array}$ & $\begin{array}{l}1.2 \\
2.5\end{array}$ & $\begin{array}{l}3.8 \\
6.0\end{array}$ & $\begin{array}{l}2.4 \\
4.7\end{array}$ & 2.4 & $\begin{array}{l}1.8 \\
3.1\end{array}$ & $\begin{array}{l}4.9 \\
5.4\end{array}$ & $\begin{array}{l}4.2 \\
4.5\end{array}$ & $\begin{array}{r}\text { 7. } 2 \\
12.2\end{array}$ & 18.1 & 5.4 & \\
\hline & & & & 9.0 & g 17.9 & 2. 9 & 4.7 & 28.9 & 12. 9 & 4.8 & 13. 8 & 4.1 & $\begin{array}{l}3.0 \\
4.3\end{array}$ & 4.9 & $\begin{array}{l}0.4 \\
4.6\end{array}$ & $\begin{array}{l}4.5 \\
7.7\end{array}$ & $\begin{array}{l}12.3 \\
\mathrm{~d} 6.0\end{array}$ & $\begin{array}{l}10.4 \\
15.0\end{array}$ & 13. 5 & \\
\hline & & & & 2. 0 & 6.4 & 1.4 & 1.2 & 2.5 & 1.8 & & 4.3 & a 0.8 & 2.4 & 1.3 & 14.0 & 7. 2 & 7.7 & 7.4 & & \\
\hline & & & & 5.4 & 14.6 & 2. 0 & 1.2 & 8.2 & 7.4 & 2. 0 & 11.6 & 3.3 & 2.6 & 3.6 & 24.1 & 9.7 & 13.9 & d 27.4 & & \\
\hline$P_{\ldots}$. & 1932 & 5.05 percent chromium & Pipe ... & 7.4 & 10.7 & 2.4 & 1.6 & 18.0 & 11.7 & 2.3 & 2. 9 & 5.0 & 3.4 & 4. 2 & 29.6 & 13.4 & 13.0 & 7.5 & & \\
\hline & & steel. & & 9.3 & & 2. 2 & 1.9 & 23.6 & 13. 9 & 2.8 & 15.6 & 5.1 & 4. 6 & d 5.8 & $\mathrm{D}$ & 10.9 & 18.3 & 27.9 & & \\
\hline & & & & 14.3 & e 19.7 & 2.9 & 2.3 & 19.1 & 20.6 & e 3.4 & 27.4 & 9.1 & 6.1 & 10.0 & D & 11.9 & $\mathrm{D}$ & 17.5 & & \\
\hline & & & & 2.1 & 6.6 & 0.9 & 0.7 & 9.4 & 4.1 & 0.4 & 4.5 & 0.6 & 0.7 & 1.8 & 4.5 & 2.3 & 5.8 & 17.1 & 4. 9 & \\
\hline D.... & 1937 & 5.02 percent chromium & Plate & 4. 0 & & 1.3 & 1.1 & 17.9 & 5.9 & .9 & 6.6 & 1.6 & 1. 2 & 3.8 & 4.4 & 2. 2 & 12.1 & d 18.1 & $\mathrm{~d} 10.0$ & \\
\hline & & & & 9.0 & g 17.6 & 2. 0 & 1.7 & 28.8 & 7.5 & 3.0 & 14.8 & 1.1 & 1.8 & 5.6 & 4.4 & 3.6 & d 3.5 & 12. 2 & 19.1 & \\
\hline & & & & 2.1 & 7.1 & 0.9 & 0.6 & 9.6 & 3.9 & 0.4 & 4. 1 & 0.9 & 0.7 & 1.7 & 4. 2 & 2. 5 & 7.9 & 12. 2 & 5. 2 & \\
\hline $\mathrm{E}_{\ldots-}$ & 1937 & 4.67 percent chromium & $-\mathrm{do}_{\ldots} \ldots$ & 4. 0 & & 1.6 & 1.2 & 16.3 & 7.2 & .8 & 6.2 & 1.8 & 1.1 & 5.2 & 5. 2 & 2.1 & 12.0 & ${ }^{d} 11.7$ & 9.9 & \\
\hline & & steel with Mo. & & 9. 0 & g 16.2 & 2.0 & 1.7 & 28.2 & 6.4 & 2. 5 & 13. 2 & 1.4 & 2. 0 & 5.3 & 4.2 & 3.3 & d 5.2 & 11. 2 & 19. 5 & \\
\hline & & & & 2.1 & b 8.3 & b 0.8 & 0.7 & 11.4 & 3.5 & 0.2 & 4.6 & 0.8 & 0.7 & b 1.7 & 5.9 & 2. 5 & 8.4 & 12.6 & 5. 0 & \\
\hline $\mathrm{H} \ldots . . .$. & 1937 & 5.76 percent chromium & -.-.do_...- & 4. 0 & & 1.4 & 1.1 & 14.3 & b 6.8 & .7 & 6.2 & 1.6 & 1.3 & 5.4 & 5.0 & 2. 2 & 14.5 & d 12.9 & 10.2 & \\
\hline & & steel with Mo. & & 9.0 & g 16.7 & 1.9 & 2.0 & & 6.1 & 2.2 & 14.2 & b 1.0 & 1.9 & 6.4 & 4.0 & 3.7 & d 5.0 & 8. 9 & 17.6 & \\
\hline
\end{tabular}

a Exposed for 1.0 yr only.

b Data for 1 specimen. The other specimen was missing.

D, both specimens destroyed by corrosion.

- Data for 1 specimen. The other specimen was destroyed by corrosion.

d Data for the individual specimens differed from the average by more than $50 \%$.

g Data for 8 specimens.

- Data for 4 specimens.

ispecimens composed of 3 layers as follows: outer layers-plain carbon steel, 0.036 in. thick; intermediate layer-2\% nickel-1\% copper steel, 0.100 in. thick. 


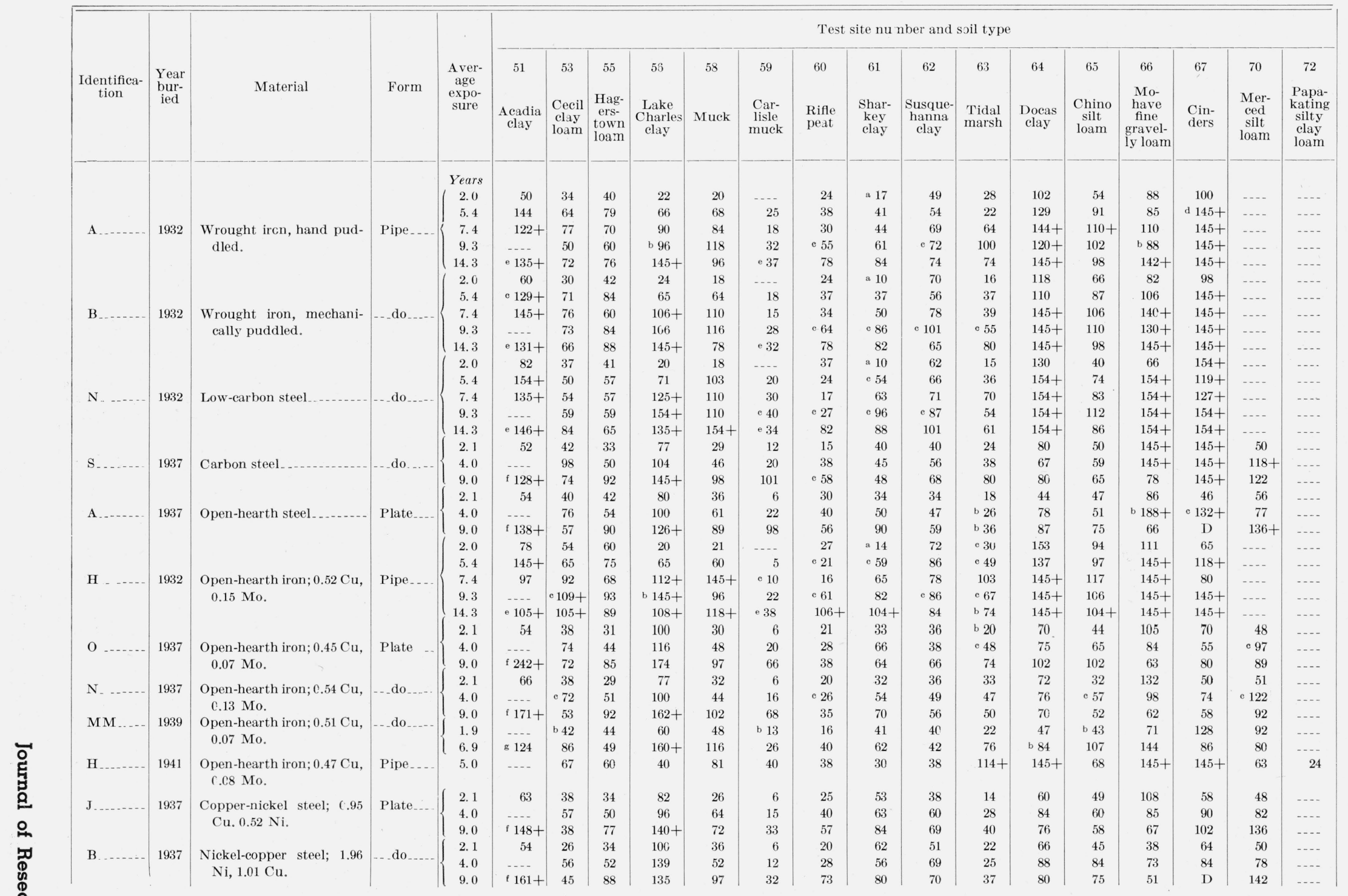




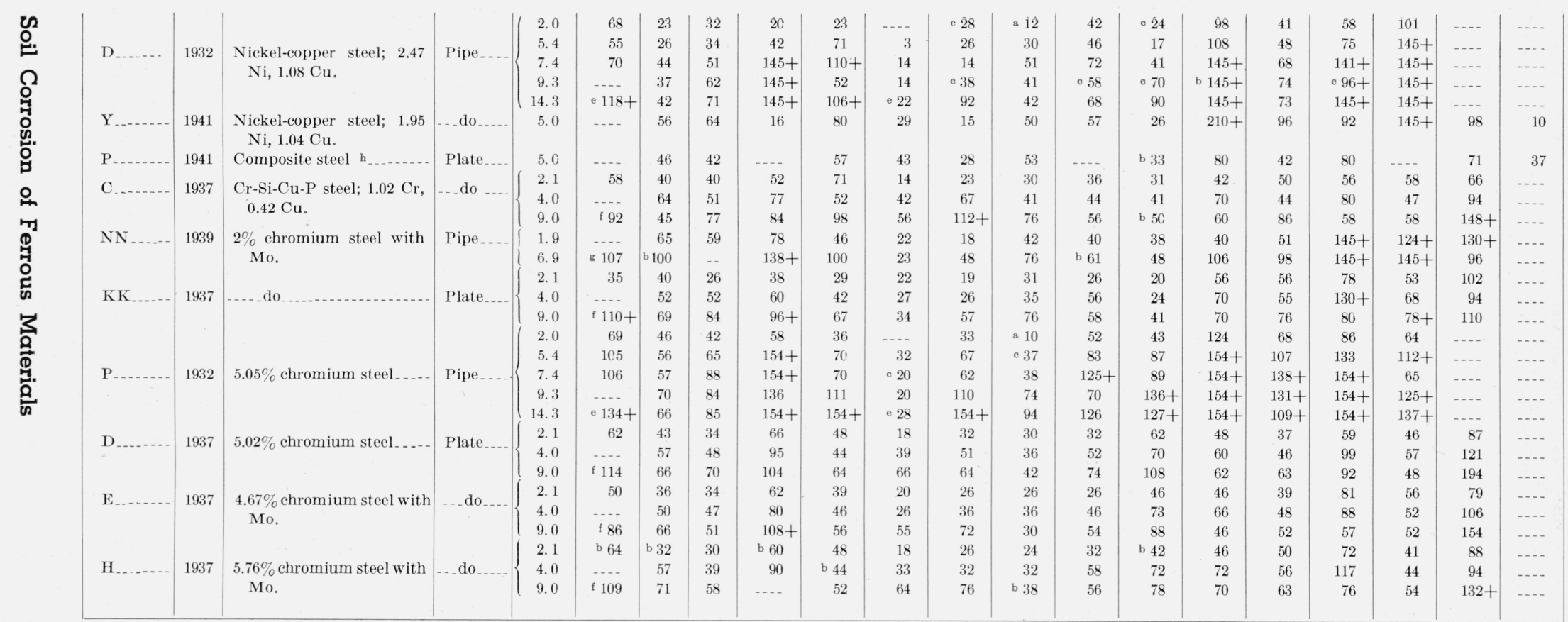

a Exposed for 1.0 yr only.

b Data for 1 specimen. The other specimen was missing.

- Data for the individual specimens differed from the average by more than $50 \%$.

$\mathrm{d}+$, one or more specimens contained holes because of corrosion.

e Data for 4 specimens.

g Data for 10 specimens.

h Specimens composed of 3 layers as follows: outer layers-plain carbon steel, 0.036 in. thick; intermediate layer-2\% nickel, $1 \%$ copper, 0.100 in. thick. 
In order to compare the over-all behavior of the materials and also to observe the effect of various alloying elements on corrosion, the data reported in tables 5 and 6 for each material at all of the test sites were averaged and shown graphically as weight loss and pit depth-time curves for the pipe specimens exposed $14 \mathrm{yr}$ (fig. 3) and for the plate specimens exposed 9 yr (fig. 4). For comparison with the low-alloy materials in the form of plates, data for two reference materials, exposed simultaneously with the low-alloy wrought materials, are included in figure 4, namely, open-hearth iron A, and carbon steel S. Specimens of the latter material were in the form of pipe.

Although the curves shown in figure 3 indicate differences in the corrosion of certain of the materials, it is impossible to state without statistical analysis of the data whether certain of these differences at least should not be ascribed to

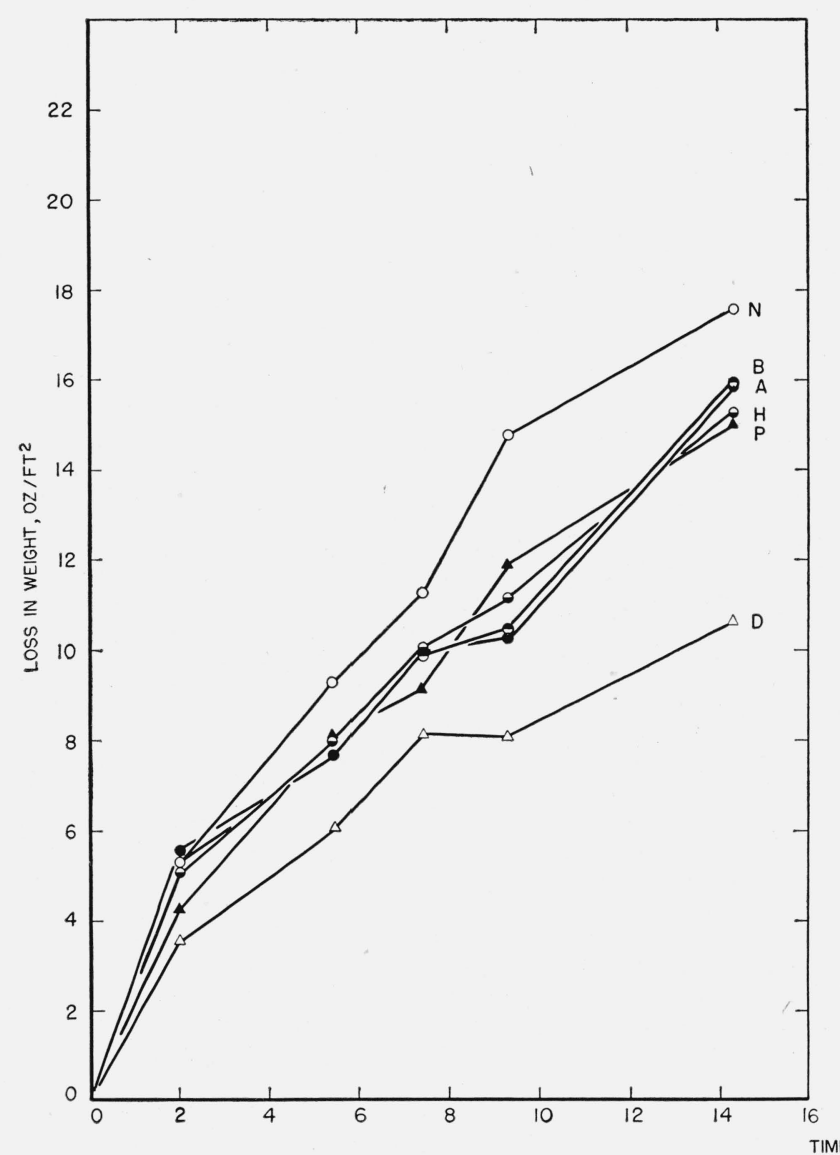

chance causes rather to inherent differences in corrodibility of the materials. As the first step in analyzing the data statistically, the progress of weight loss and pitting of each material with time was expressed by means of equations of the form

$$
y=a x^{n} .
$$

Taking logarithms of both sides, this equation takes the form of the linear equation

$$
\log y=\log a+n \log x .
$$

Expressing the logarithm of the pit depth as $\log$ $P$, the logarithm of the time as $\log T$, and the $y$-intercept as $\log k$, the following equation is obtained:

$$
\log P=\log k+n \log T
$$

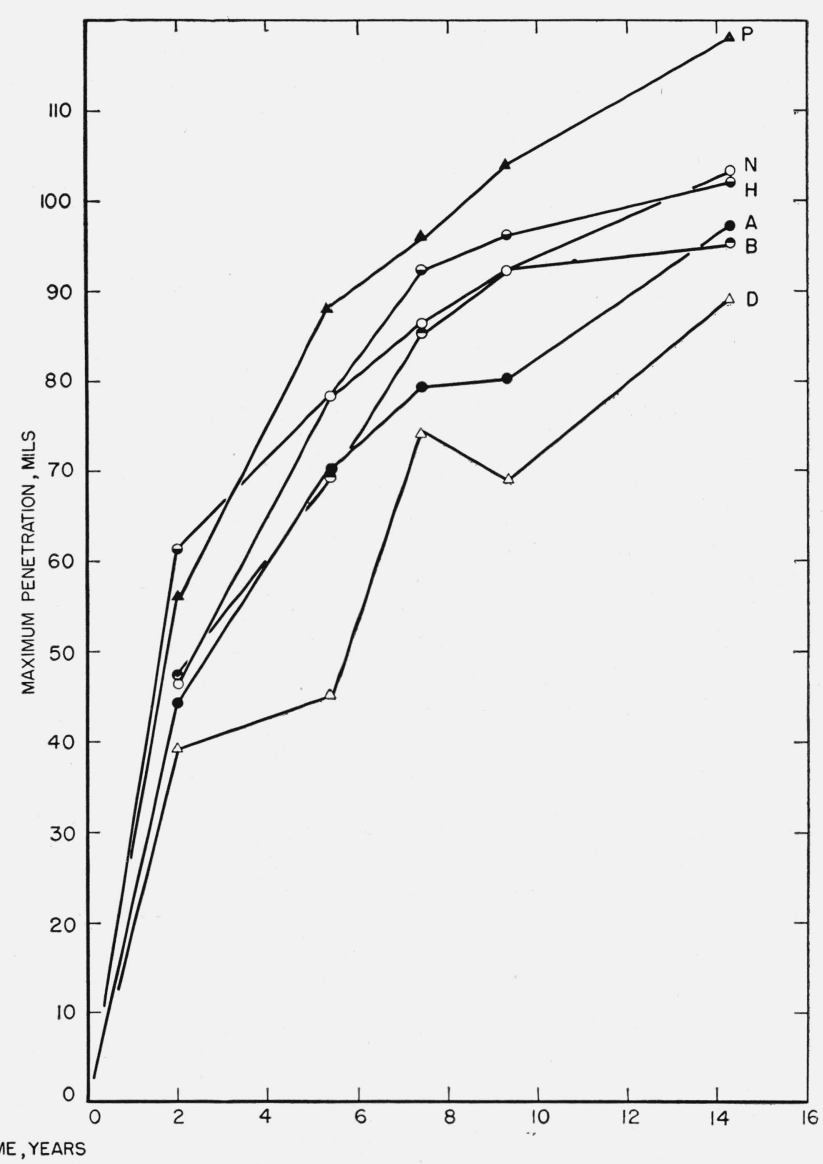

Figure 3. Average loss in weight and maximum penetration-time curves of wrought pipe specimens in 13 soils.

A, Wrought iron, hand-puddled; B, wrought iron, mechanically puddled; H, open hearth iron 0.52-percent Cu, 0.15-percent Mo; N, low carbon steel; D, steel, 2.47-percent Ni, 1.01-percent Cu; P, steel, 5.05-percent Cr. 

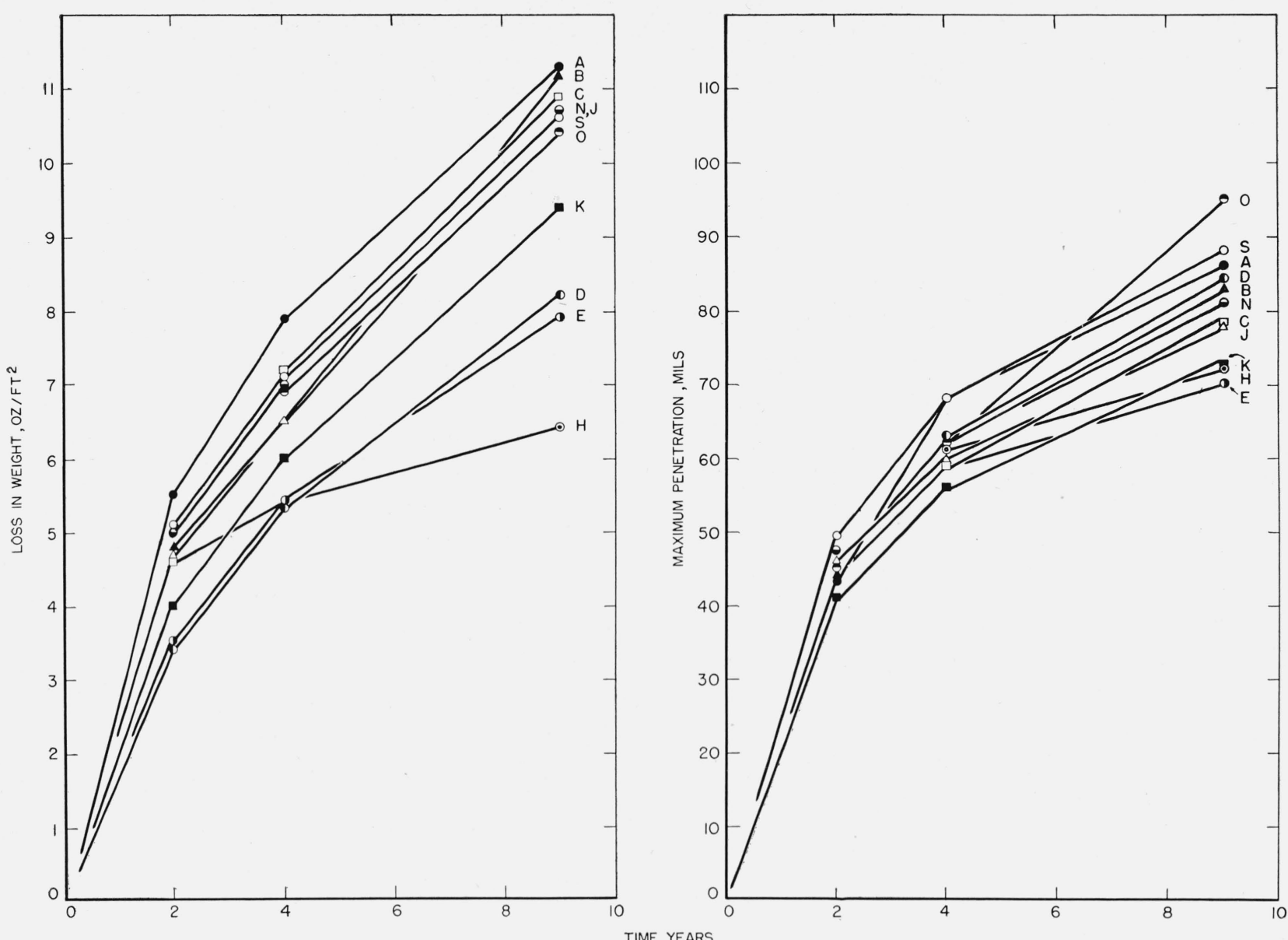

Figure 4. Average loss in weight and maximum penetration-time curves of wrought plate specimens in 14 soils.

O, Open-hearth iron, 0.45-percent $\mathrm{Cu}, 0.07$-percent $\mathrm{Mo}$; N, open-hearth iron, 0.54-percent $\mathrm{Cu}, 0.13$-percent Mo; $\mathrm{S}$, plain carbon steel; A, open-hearth steel; J, steel, 0.95-percent $\mathrm{Cu}$, 0.52-percent Ni; B, steel, 1.96-percent Ni, 1.01-percent Cu; C, Cr-Si-Cu-P steel-1.02-percent Cr, 0.42-percent Cu; K, steel, 5.02-percent Cr; E, steel, 4.67-percent Cr, 0.51-percent Mo; H, steel, 5.76-percent Cr, 0.43-percent Mo.

By similar substitutions, a linear equation expressing the relation between weight loss $W$, and time was obtained:

$$
\log W=\log k_{1}+u \log T .
$$

These equations, which were derived originally by Logan, Ewing, and Denison [3] and by Martin [4] to express, respectively, the progress with time of the pitting and weight loss of wrought ferrous metals in soils, were fitted to the data shown in figure 3 by the method of least squares. The mean values of weight loss and pit depth given in table 7 were computed by eq 3 and 4, respectively, for the period of $14 \mathrm{yr}$. Values for the standard error of these means, shown also in the table, were calculated by the method of Ezekiel [5]. In order to determine whether the various wrought materials corroded at significantly different rates from plain steel, the mean values of weight loss and pitting for each material at $14 \mathrm{yr}$ were compared with the corresponding values for plain steel, taking into account the respective standard errors. The standard errors of the difference between the means for plain steel and for each material with which it was compared were calculated by the following equation to be found in standard texts on statistical methods [6]:

$$
\begin{aligned}
\sigma_{D} & =\sqrt{\sigma_{\bar{x}_{1}}^{2}+\sigma_{\bar{x}_{2}}^{2}} \\
& =\sqrt{\frac{\sigma_{1}^{2}}{N_{1}}+\frac{\sigma_{2}^{2}}{N_{2}}},
\end{aligned}
$$


TABLE 7. Comparison of average corrosion at all test sites of wrought materials exposed 14 years

\begin{tabular}{|c|c|c|c|c|c|c|c|c|c|c|c|}
\hline \multirow[b]{2}{*}{$\begin{array}{l}\text { Identi- } \\
\text { fica- } \\
\text { tion }\end{array}$} & \multirow[b]{2}{*}{ Material } & \multicolumn{5}{|c|}{ Loss in weight (oz/ft²) } & \multicolumn{5}{|c|}{ Maximum pit depth (mils) } \\
\hline & & 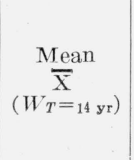 & $\begin{array}{l}\text { Stand- } \\
\text { ard } \\
\text { error } \\
\bar{\sigma}\end{array}$ & $\begin{array}{l}\text { Differ- } \\
\text { ence of } \\
\text { the mean } \\
\text { from } \\
\text { material } \\
N \quad \\
\quad D\end{array}$ & $\begin{array}{c}\text { Standard } \\
\text { error of } \\
\text { the dif- } \\
\text { ference a } \\
\sigma_{D}\end{array}$ & $2 \sigma_{D}$ & $\begin{array}{c}\text { Mean } \\
\overline{\mathrm{X}} \\
\left(P_{T}=14 \mathrm{yr}\right)\end{array}$ & $\begin{array}{l}\text { Stand- } \\
\text { ard } \\
\text { error } \\
\bar{\sigma}\end{array}$ & $\begin{array}{c}\text { Differ- } \\
\text { ence of } \\
\text { the mean } \\
\text { from } \\
\text { material } \\
N \quad \\
\quad D\end{array}$ & $\begin{array}{l}\text { Standard } \\
\text { error of } \\
\text { the dif- } \\
\text { ference a } \\
\sigma_{D}\end{array}$ & $2 \sigma_{D}$ \\
\hline $\mathrm{N}$ & Plain steel & 16. 7 & 1.4 & ....... & ....... & & 113 & 4. 0 & & & .... \\
\hline A & Hand-puddled wrought iron............ & 14.8 & 1.2 & -1.9 & 1.8 & 3.6 & 103 & 4.1 & -10 & 5.7 & 11.4 \\
\hline $\mathrm{B}$ & Mechanically puddled wrought iron... & 14.3 & 1.2 & -2.4 & 1.8 & 3.6 & 107 & 5.5 & -6 & 6.9 & 13.8 \\
\hline $\mathrm{H}$ & Open-hearth iron & 13.6 & 1.0 & -3.1 & 1.7 & 3.4 & 109 & 1.5 & -4 & 4.3 & 8.6 \\
\hline $\mathrm{P}$ & $5 \% \mathrm{Cr}$ steel & 13.5 & 1.2 & -3.2 & 1.8 & 3.6 & 125 & 5.0 & +12 & 6.4 & 12.8 \\
\hline $\mathrm{D}$ & $2 \% \mathrm{Ni}-1 \%$ Cu steel & 10.7 & 1.1 & -6.0 & 1.8 & 3.6 & 92.2 & 8.3 & -20.8 & 9.1 & 18.2 \\
\hline
\end{tabular}

where

$\sigma_{1}=$ standard deviation of first sample,

$\sigma_{2}=$ standard deviation of second sample,

$N_{1}=$ number of observations of first sample,

$N_{2}=$ number of observations of second sample.

By comparing the standard error of the difference $\sigma_{D}$ with the difference between the two means $\bar{X}_{1}$ and $\bar{X}_{2}$, the probability that the observed difference is significant can be found by reference to tables of probability corresponding to the number of times the observed difference exceeds the standard error. For the present study, a difference between means of at least twice the standard error was selected. A difference of this magnitude. corresponds to a probability of 21 to 1 that the observed difference is significant.

On the basis of this criterion, only material $D$, containing 2 percent of nickel and 1 percent of copper, can be considered definitely to be more resistant to weight loss and to pitting than the plain steel, $N$. However, there is some probability that material $P$, containing 5 percent of chromium, is slightly more resistant than plain steel with respect to weight loss, but less resistant to pitting. The small differences shown by the other materials as compared with plain steel are probably to be ascribed to chance causes.

The weight loss data shown in figure 4 for the plate specimens exposed for a maximum of $9 \mathrm{yr}$ indicate that all of the materials except those containing 2 percent or more of chromium, namely, materials $K, D, E$, and $H$, corroded essentially alike. The beneficial effect of chromium in higher concentration is indicated by the lower average weight losses for the 5-percent chromium steel $D$.
The effect of molybdenum in reducing the corrosion of chromium steels is indicated by the curves for materials $E$ and $H$, both of which contain approximately 0.5 percent of molybdenum in addition to 4.67 and 5.76 percent of chromium, respectively.

The benefit conferred by chromium in reducing weight loss apparently does not extend to maximum penetration, because the 5-percent chromium steel $D$, which showed definitely lower weight losses than the materials with smaller amounts of chromium, showed no improvement with respect to pitting. On the other hand, the effect of molybdenum in reducing the depth of the deepest pits as well as weight loss is indicated by the fact that the materials containing approximately 0.5 percent of molybdenum, $E, H$, and $K$, also show relatively low values for maximum penetration. As a matter of fact, the average depth of the deepest pits on material $K$, which contained only 2 percent of chromium was no greater than the corresponding values for materials $E$ and $H$, both of which contained from two to three times as much chromium.

Although the specific effect of chromium on the maximum penetration of the irons and steels under consideration is somewhat difficult to evaluate because most of the materials also contain molybdenum, there was no reason to predict that chromium in the range of 4 to 6 percent might accelerate pitting, such as is indicated by the data for material $P$ shown in figure 3 and in table 7 . In view of the possibility that chromium carbides precipitated along grain boundaries might have served as local centers of attack and so might have 

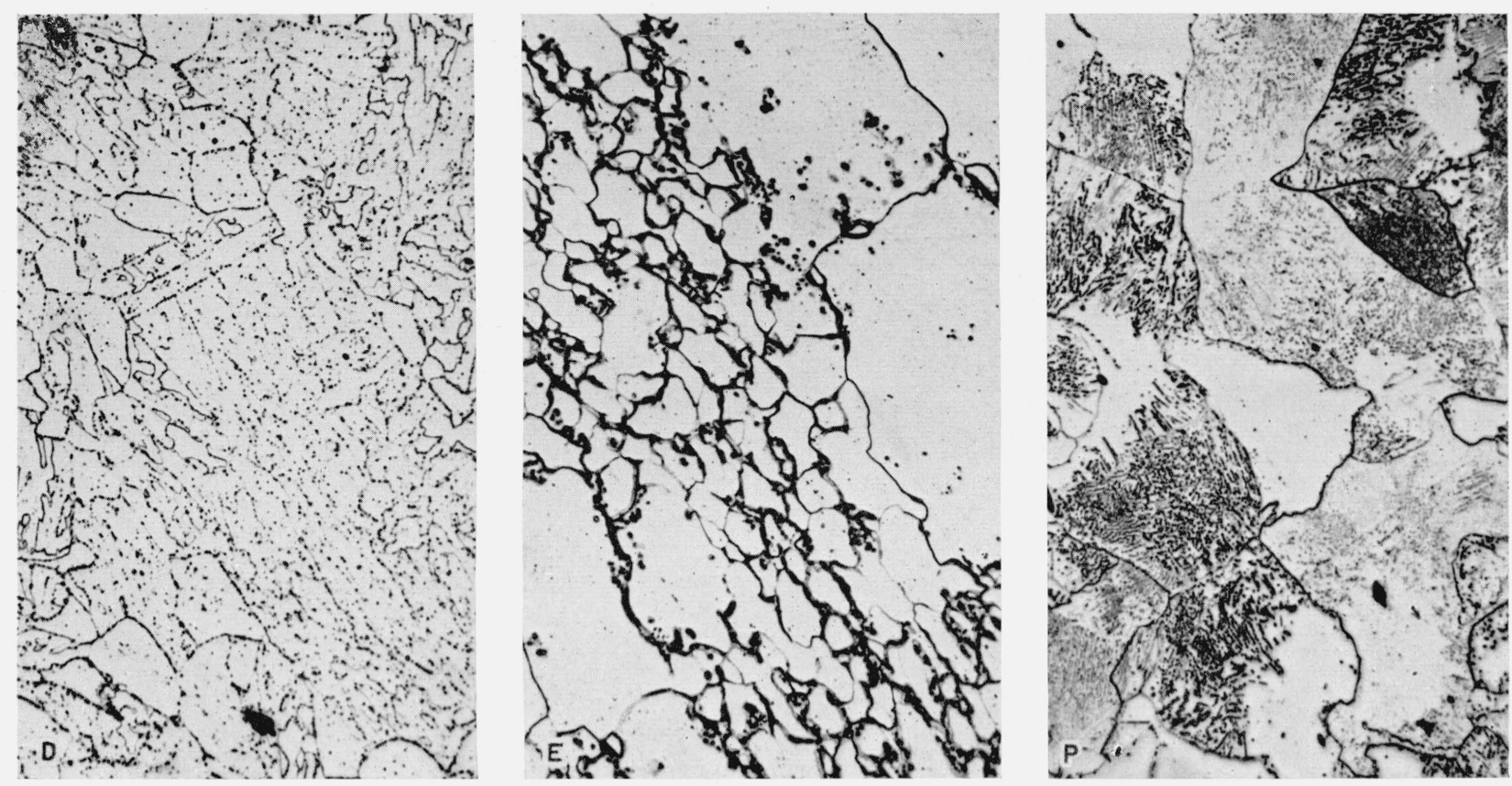

Figure 5. Longitudinal sections of 4-6 percent chromium steel specimens.

500

D, 5.02-percent chromium; E, 4.67-percent chromium with 0.51-percent molybdenum; P, 5.05-percent chromium.

accelerated pitting, microphotographs of material $P$ were prepared for comparison with chromium steels that were considered to show normal behavior.

Microscopical examination of steel $P$ (fig. 5) reveals that the carbides are in lamellar form, partly spheroidized. By contrast, the carbides of steel $D$ are completely spheroidized and are completely dispersed throughout the matrix. Because neither of these steels contains molybdenum, the carbides are probably chromium carbides. As the microstructure of material $P$ is considered to be that of a normal 4- to 6-percent chromium steel, the apparent accelerated pitting of this material cannot at present be accounted for.

The carbides of steel $E$ (fig. 5) are localized around the grain boundaries. Because this steel contains an appreciable amount of molybdenum most of the carbon was probably combined with molbydenum, and the chromium remained in solution in the ferrite. This may account for the slightly improved resistance of steel $E$ as compared with steel $D$.

The results of the tests of steels $B$ and $D$, containing 2 percent of nickel and 1 percent of copper, are conflicting. Material $D$ is definitely superior to plain steel with respect to weight loss and pitting (fig. 3), whereas material $B$, having the same nominal composition, shows no advantage from its content of nickel and copper. A probable explanation for this difference in behavior is the acceleration of corrosion from the presence of mill scale on material $B$, which unlike $D$, had not been pickled.

Comparison of the average corrosion resistance of the different materials in all of the soils reveals nothing concerning the behavior of these materials in any one soil or environment. Consequently, superior corrosion resistance of a material in any one environment might be obscured by inferior resistance in another. In order to compare the behavior of the materials under specific environmental conditions, the corrosion data for the soils classified according to environment (table 2) were calculated on a relative basis for each period of exposure, the standards of reference, carbon steel $(N)$ and open-hearth steel $(A)$, being taken as 100 percent. The averages for all periods and for all soils in the same environmental group are given in tables 8 and 9 . 
TABLE 8. Effect of composition on the corrosion of low-alloy iron and steel pipe specimens in different soil environments - maximum exposure 14 years

\begin{tabular}{|c|c|c|c|c|c|c|c|c|c|c|}
\hline \multirow{4}{*}{ Identification } & \multicolumn{4}{|c|}{ Composition of steel (percent) } & \multicolumn{6}{|c|}{ Environment } \\
\hline & \multirow{3}{*}{$\mathrm{Cr}$} & \multirow{3}{*}{$\mathrm{Ni}$} & \multirow{3}{*}{$\mathrm{Cu}$} & \multirow{3}{*}{ Mo } & Organic & \multicolumn{4}{|c|}{ Inorganic } & \multirow{3}{*}{ Cinders } \\
\hline & & & & & \multirow{2}{*}{$\begin{array}{l}\text { Reducing } \\
\text { acid }\end{array}$} & \multicolumn{2}{|c|}{ Reducing } & \multicolumn{2}{|c|}{ Oxidizing } & \\
\hline & & & & & & Acid & Alkaline & Acid & Alkaline & \\
\hline
\end{tabular}

AVERAGE LOSS IN WEIGHT FOR 5 PERIODS OF EXPOSURE-RELATIVE BASIS (PERCENT)

\begin{tabular}{|c|c|c|c|c|c|c|c|c|c|c|}
\hline $\mathrm{N}_{\ldots}$ & -... & & -... & -... & 100 & 100 & 100 & 100 & 100 & 100 \\
\hline D $\ldots$ & -... & 2.47 & 1.08 & -..- & 70 & 76 & 63 & 67 & 53 & 100 \\
\hline $\mathrm{H}$ & .... & .... & 0.52 & 0.15 & 78 & 96 & 78 & 94 & 104 & 81 \\
\hline P & 5.05 & .... & ...- & ...- & 75 & 94 & 81 & 62 & 94 & 59 \\
\hline
\end{tabular}

AVERAGE MAXIMUM PENETRATION FOR 5 PERIODS OF EXPOSURE-RELATIVE BASIS (PERCENT)

\begin{tabular}{|c|c|c|c|c|c|c|c|c|c|c|}
\hline$N_{\ldots} \ldots$ & -... & -... & .... & ...- & 100 & 100 & 100 & 100 & 100 & 100 \\
\hline D $\ldots \ldots$ & $\ldots$ & 2.47 & 1.08 & -... & 85 & 66 & 90 & 75 & 78 & 99 \\
\hline $\mathrm{H}_{\ldots \ldots \ldots}$ & $\ldots$ & -... & 0.52 & 0.15 & 103 & 97 & 94 & 132 & 127 & 80 \\
\hline$P_{\ldots} \ldots$ & 5.05 & .... & .... & ....- & 156 & 82 & 132 & 118 & 124 & 74 \\
\hline
\end{tabular}

TABLE 9. Effect of composition on the corrosion of low-alloy iron and steel plate specimens in different soil environments-maximum exposure 9 years

\begin{tabular}{|c|c|c|c|c|c|c|c|c|c|c|}
\hline \multirow{4}{*}{ Identification } & \multicolumn{4}{|c|}{ Composition of steel (percent) } & \multicolumn{6}{|c|}{ Environment } \\
\hline & \multirow{3}{*}{$\mathrm{Cr}$} & \multirow{3}{*}{$\mathrm{Ni}$} & \multirow{3}{*}{$\mathrm{Cu}$} & \multirow{3}{*}{ Mo } & Organic & \multicolumn{4}{|c|}{ Inorganic } & \multirow{3}{*}{ Cinders } \\
\hline & & & & & \multirow{2}{*}{$\begin{array}{c}\text { Reducing } \\
\text { acid }\end{array}$} & \multicolumn{2}{|c|}{ Reducing } & \multicolumn{2}{|c|}{ Oxidizing } & \\
\hline & & & & & & Acid & Alkaline & Acid & Alkaline & \\
\hline
\end{tabular}

AVERAGE LOSS IN WEIGHT FOR 3 PERIODS OF EXPOSURE-RELATIVE BASIS (PERCENT)

\begin{tabular}{|c|c|c|c|c|c|c|c|c|c|c|}
\hline A $\ldots \ldots$ & 0.049 & 0.034 & 0.052 & $\ldots$ & 100 & 100 & 100 & 100 & 100 & 100 \\
\hline $\mathrm{O}_{\ldots} \ldots$ & .02 & .15 & .45 & 0.07 & 83 & 98 & 94 & 104 & 93 & 133 \\
\hline$N_{\ldots} \ldots \ldots$ & .02 & .14 & .54 & .13 & 82 & 94 & 96 & 102 & 92 & 102 \\
\hline $\mathrm{J}_{\ldots} \ldots \ldots$ & $-\ldots$ & .52 & .95 & -..- & 79 & 87 & 96 & 73 & 87 & 124 \\
\hline B $\ldots$ & -..- & 1. 96 & 1. 01 & $\ldots$ & 90 & 82 & 93 & 59 & 91 & 145 \\
\hline C $\ldots$ & 1. 02 & .22 & .428 & $\ldots$ & 85 & 90 & 88 & 80 & 102 & 84 \\
\hline $\mathrm{K} \ldots$ & 2.01 & .07 & .004 & .57 & 62 & 88 & 80 & 89 & 91 & 71 \\
\hline D _... & 5. 02 & .09 & .008 & $\ldots$ & 52 & 53 & 85 & 41 & 60 & 68 \\
\hline E $\ldots$ & 4. 67 & .09 & .004 & .51 & 51 & 54 & 85 & 43 & 61 & 51 \\
\hline $\mathrm{H}_{\ldots} \ldots \ldots \ldots$ & 5. 76 & .17 & .004 & .43 & 52 & 54 & 76 & 42 & 72 & 50 \\
\hline
\end{tabular}

AVERAGE MAXIMUM PENETRATION FOR 3 PERIODS OF EXPOSURE-RELATIVE BASIS (PERCENT)

\begin{tabular}{|c|c|c|c|c|c|c|c|c|c|c|}
\hline A $\ldots$ & 0.049 & 0.034 & 0.052 & $\ldots$ & 100 & 100 & 100 & 100 & 100 & 100 \\
\hline $\mathrm{O}_{\ldots} \ldots \ldots$ & .02 & .15 & .45 & 0.07 & 102 & 123 & 110 & 97 & 97 & 62 \\
\hline $\mathrm{N} \ldots \ldots$ & .02 & .14 & .54 & .13 & 97 & 106 & 104 & 95 & 84 & 56 \\
\hline$J_{\ldots} \ldots$ & .... & .52 & .95 & $\ldots$ & 81 & 113 & 102 & 93 & 86 & 76 \\
\hline B $\ldots \ldots$ & $\ldots$ & 1. 96 & 1. 01 & $\ldots$ & 85 & 113 & 111 & 100 & 82 & 91 \\
\hline C $\ldots \ldots$ & 1.02 & 0.22 & 0.428 & $\ldots$ & 130 & 81 & 88 & 91 & 80 & 50 \\
\hline K & 2.01 & .07 & .004 & .57 & 82 & 78 & 90 & 93 & 96 & 60 \\
\hline $\mathrm{D}$ & 5.02 & .09 & .008 & $\cdots$ & 149 & 77 & 105 & 97 & 76 & 46 \\
\hline $\mathrm{E}_{\ldots} \ldots \ldots$ & 4. 67 & .09 & .004 & .51 & 130 & 62 & 94 & 83 & 72 & 49 \\
\hline $\mathrm{H}_{\ldots} \ldots$ & 5. 76 & .17 & .004 & .43 & 128 & 72 & 94 & 88 & 87 & 42 \\
\hline
\end{tabular}


Additions of 0.5 percent of copper and 0.15 percent of molybdenum are seen from the data reported in table 8 (material $H$ ) and in table 9, materials $O$ and $N$, to have had no significant influence on the rate of corrosion. As a matter of interest, it may be noted that the addition of copper tends to increase slightly the loss in weight of steels in sea water, but has little or no effect on pitting [7]. The inconsistent behavior of materials $B$ and $D$, each containing 2 percent of nickel and 1 percent of copper, has been previously commented upon.

As was previously noted, the effects of additions of chromium are difficult to evaluate because most of these steels also contain molybdenum. All of the chromium steels lost less weight than the reference material $A$ (table 9). The maximum penetration is also less for all environments except the organic-reducing. This benefit of chromium is especially marked in cinders. In general, the maximum resistance to corrosion is shown by steel
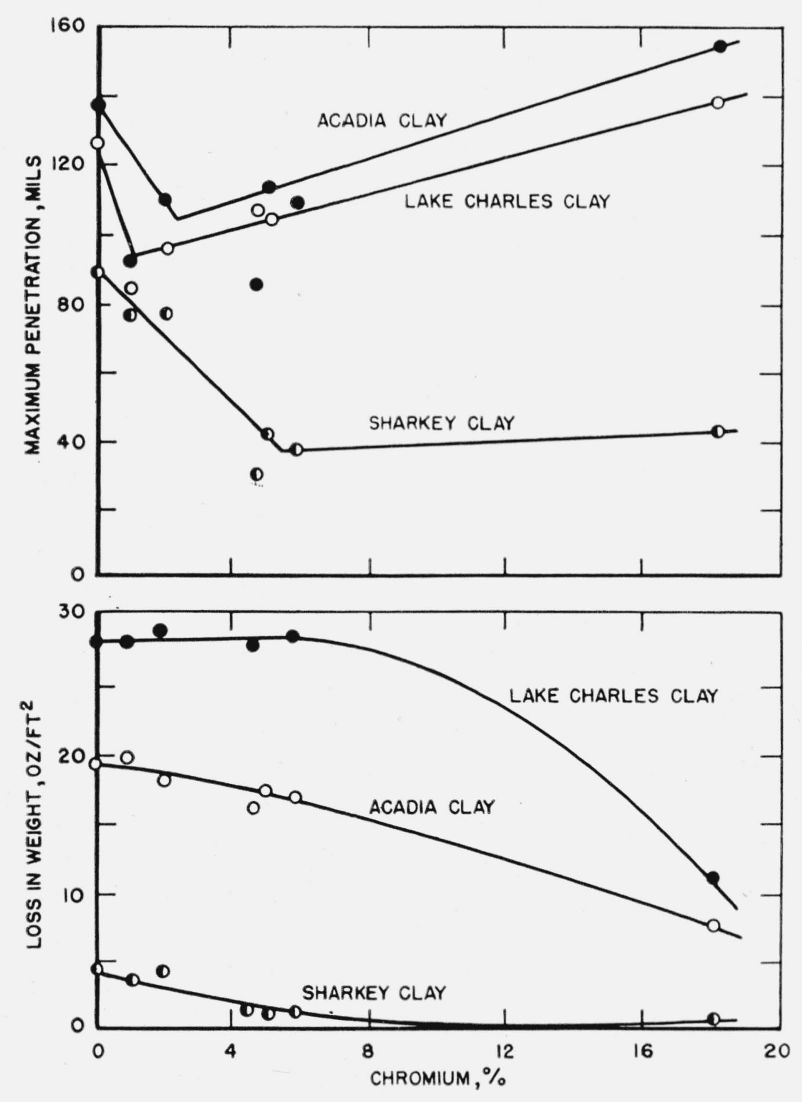

Eigure 6." Effect of chromium content on loss in weight and pitting of steels in three poorly aerated soils, exposure $9 \mathrm{yr}$.
$E$, containing slightly less than 5 percent of chromium and 0.5 percent of molybdenum.

The curves shown in figure 6 indicate that the advantage gained in reduction of pitting by the addition of small amounts of chromium may be lost if the amount added exceeds a certain optimum value. In fact, the pit depth curves for Acadia clay and Lake Charles clay in figure $6{ }^{2}$ indicate that steels containing 18 percent of chromium show a slight acceleration of pitting over plain steel. The tendency for chromium to accelerate the pitting of steels in natural waters has previously been noted; LaQue [7] cited the work of Speller on the corrosion of steels in river water, and on the basis of his own observations in sea water, concluded that addition of chromium to steels for the purpose of reducing weight losses in natural waters should probably not exceed 3 percent, because larger amounts may accelerate pitting.

\section{(b) High-Alloy Steels}

Corrosion data for the high-chromium and chromium-nickel steels, some exposed for $7 \mathrm{yr}$ and some for $14 \mathrm{yr}$, are given in table 10; and the effects of composition on the corrosion of three steels in three soils are illustrated in figure 7 . It is unfortunate that complete information concerning the rolling and heat treatment of the various steels is not available, because these factors undoubtedly played a part in the corrosion of the materials. It is shown quite conclusively, however, that plain chromium steels are subject to severe pitting, and that steels containing at least 18 percent of chromium and 8 percent of nickel are considerably more resistant than the straight chromium steels in these environments. Steels containing larger amounts of chromium and nickel, with and without molybdenum, were wholly resistant to corrosion, but two of these steels were exposed for $7 \mathrm{yr}$ only, as compared with $14 \mathrm{yr}$ for the other materials.

Because high-chromium-nickel steels are susceptible to pitting where oxygen is excluded locally as, for example, by adherent deposits in sea water, the corrosion resistance shown by some of the steels in soils deficient in oxygen and high in chlorides is noteworthy. Docas clay (soil 64)

2 The composition of the steel containing $18 \%$ of chromium was not recorded in table 3 because the complete analysis of this steel is not available. 

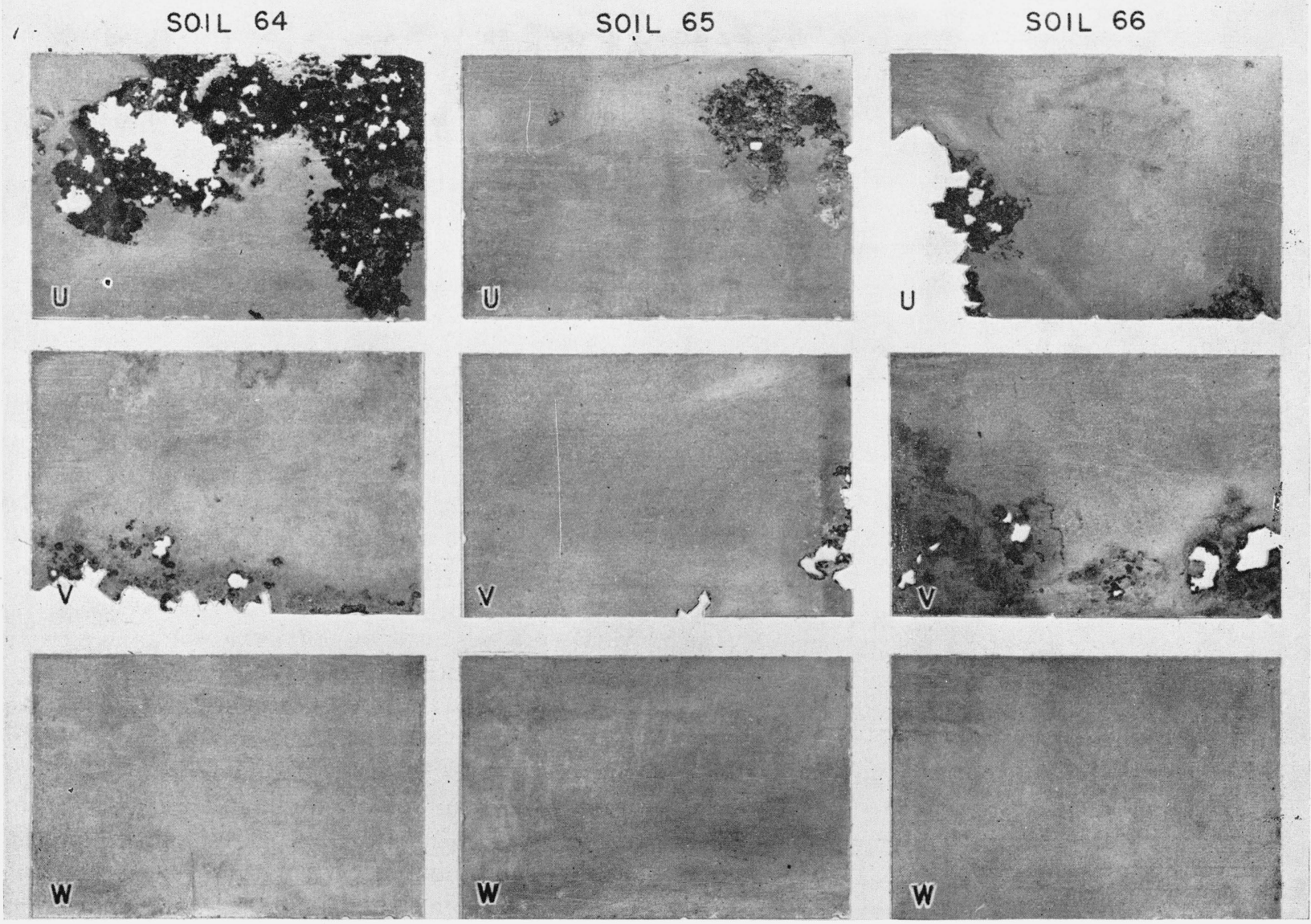

Figure 7. Specimens of high-alloy steels ajter exposure for $14 \mathrm{yr}$.

U, 12-percent-chromium steel; V, 18-percent-chromium steel; W. 18-percent-chromium, 9-percent-nickel steel. 
TABLE 10. Loss in weight and depth of pits of high chromium and chromium-nickel steels exposed for 7 years and for 14 years ${ }^{3}$

M, Shallow metal attack, roughening of surfaces, but no definite pitting. P, Definite pitting, but no pits greater than 6 mils.

+ , One or more specimens contained holes because of corrosion.

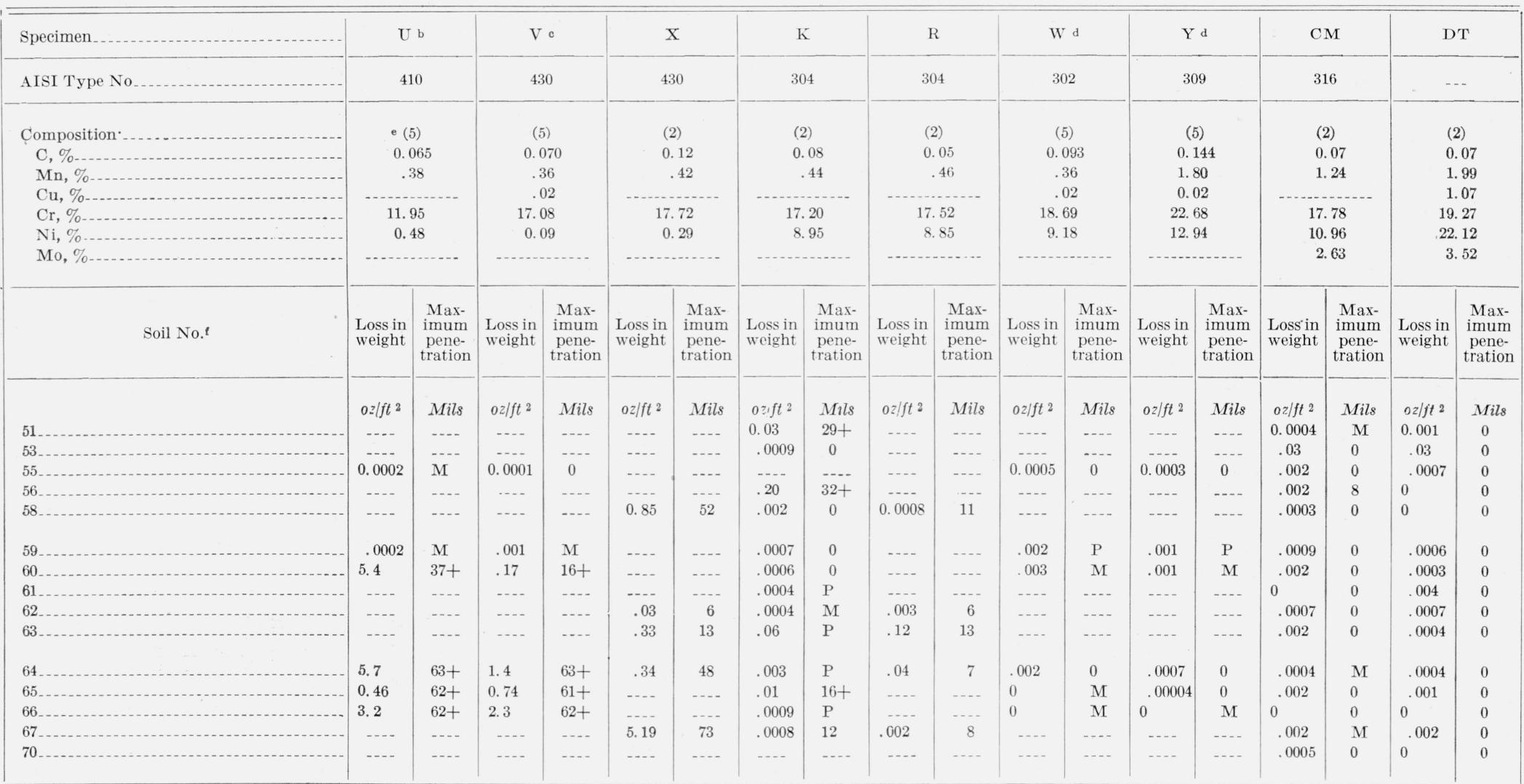

a Specimens "CM" and "DT" were exposed to the soils for $7 \mathrm{yrs}$. The remaining specimens were exposed" $14 \mathrm{yrs}$.

b Annealed at $1,400^{\circ} \mathrm{F}$, pickled, lightly cold-rolled.

- Annealed at 1,600 F, pickled.

¿ Heated to $1,850^{\circ} \mathrm{F}$, air quenched, pickled.

- The numbers in parentheses indicate the number of specimens removed from each test site.

See table 1 for names and location of soils. 
TABLE 11. Results of exposure of high-alloy steels to a poorly aerated soil containing sodium chloride (Docas clay, soil 64)

M, Shallow metal attack, roughening at surface, but no definite pitting.

+ , One or more specimens contained holes because of corrosion. The thickness of the specimen has been used as the maximum pit in this case.

D, Destroyed by corrosion.

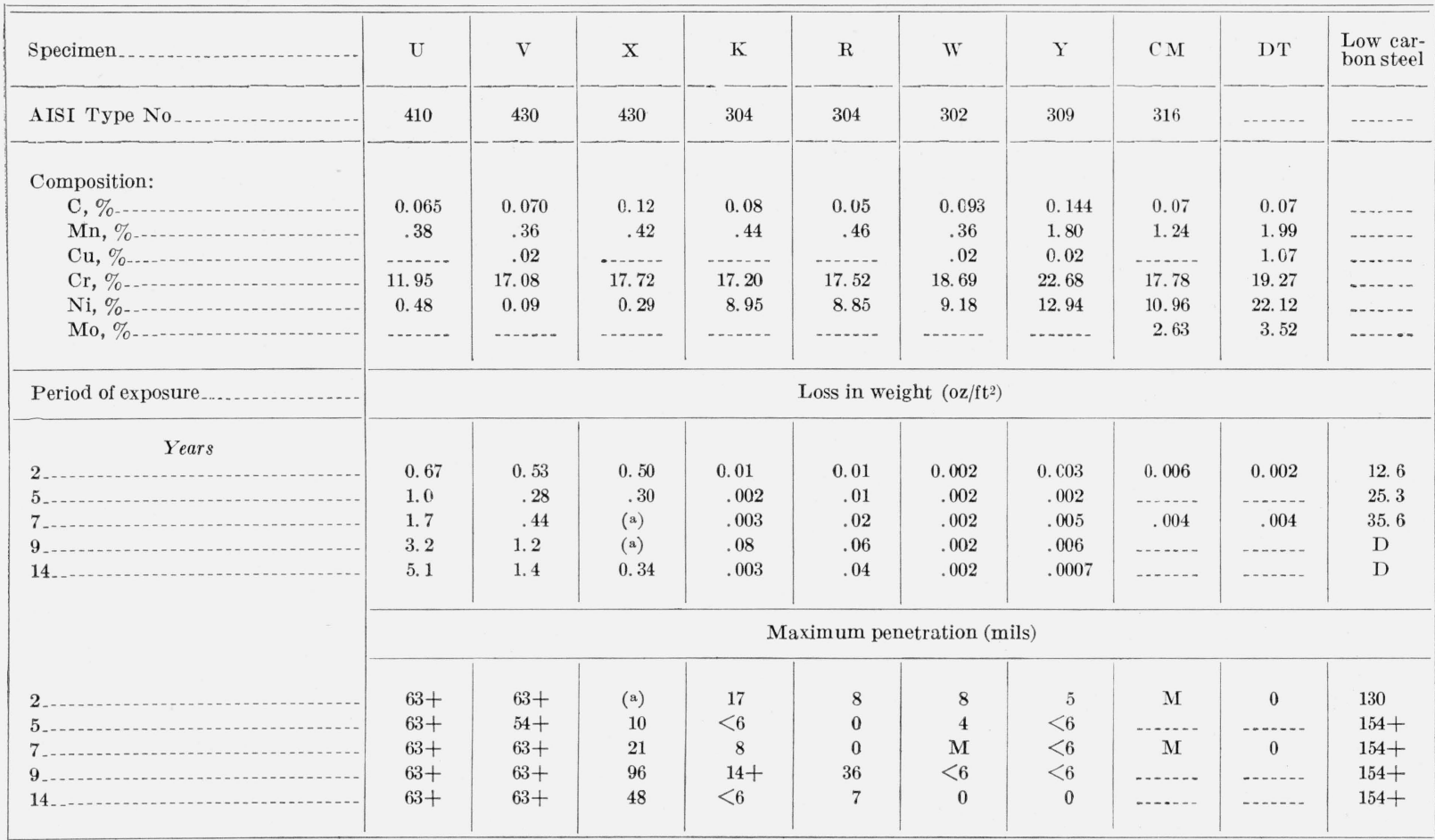

a Data not used because of corrosion caused by asphalt on the ends of the specimens.

is a very corrosive soil because of its low permeability to air and its high content of sodium chloride. The results of the field tests in this soil, given in table 11 for all periods, indicate the beneficial efiect of nickel in promoting corrosion resistance of these steels. Steels containing chromium but less than 0.5 percent of nickel pitted deeply, perforations occurring within 2 yrs; but steels containing more than 9 percent of nickel developed only shallow pits, which showed no tendency to increase with time.

\section{(c) Cast Materials}

The weight losses and maximum depths of pits of cast iron after exposure are given in tables 12 and 13, respectively. Following the procedure employed in studying the corrosion of the wrought specimens, the data for weight loss and pitting for each of the cast irons were averaged for all of the soils for each period of exposure. From the results for the pipe specimens buried in 1932, shown as weight loss and pit depth-time curves in figure 8 , it is evident that weight loss and maximum penetration for all materials were approximately the same for each period except for alloy $E$, which corroded considerably less than the other materials. The corrosion data shown graphically in figure 9 for the series of nickel and nickel-copper irons exposed in 1941 does not indicate any significant improvement from the additions of these alloying elements.

\section{(2) Effect of Environment}

Photographs of corroded specimens of unalloyed steel pipe $N$ and of plain cast iron pipe $G$ after exposure for $14 \mathrm{yr}$ in the various soils are shown in figures 10 and 11, respectively. Photographs of open-hearth iron plates $A$ exposed for $9 \mathrm{yr}$ at 15 test sites are shown in figure 12 .

Typical corrosion-time curves for the wrought materials that behaved essentially like plain steel are shown in figure 13. Each point on these curves is the average weight loss or maximum penetration of two specimens of each of the following materials: low carbon steel $N$, hand-puddled wrought iron $A$, mechanically puddled wrought iron $B$, and 

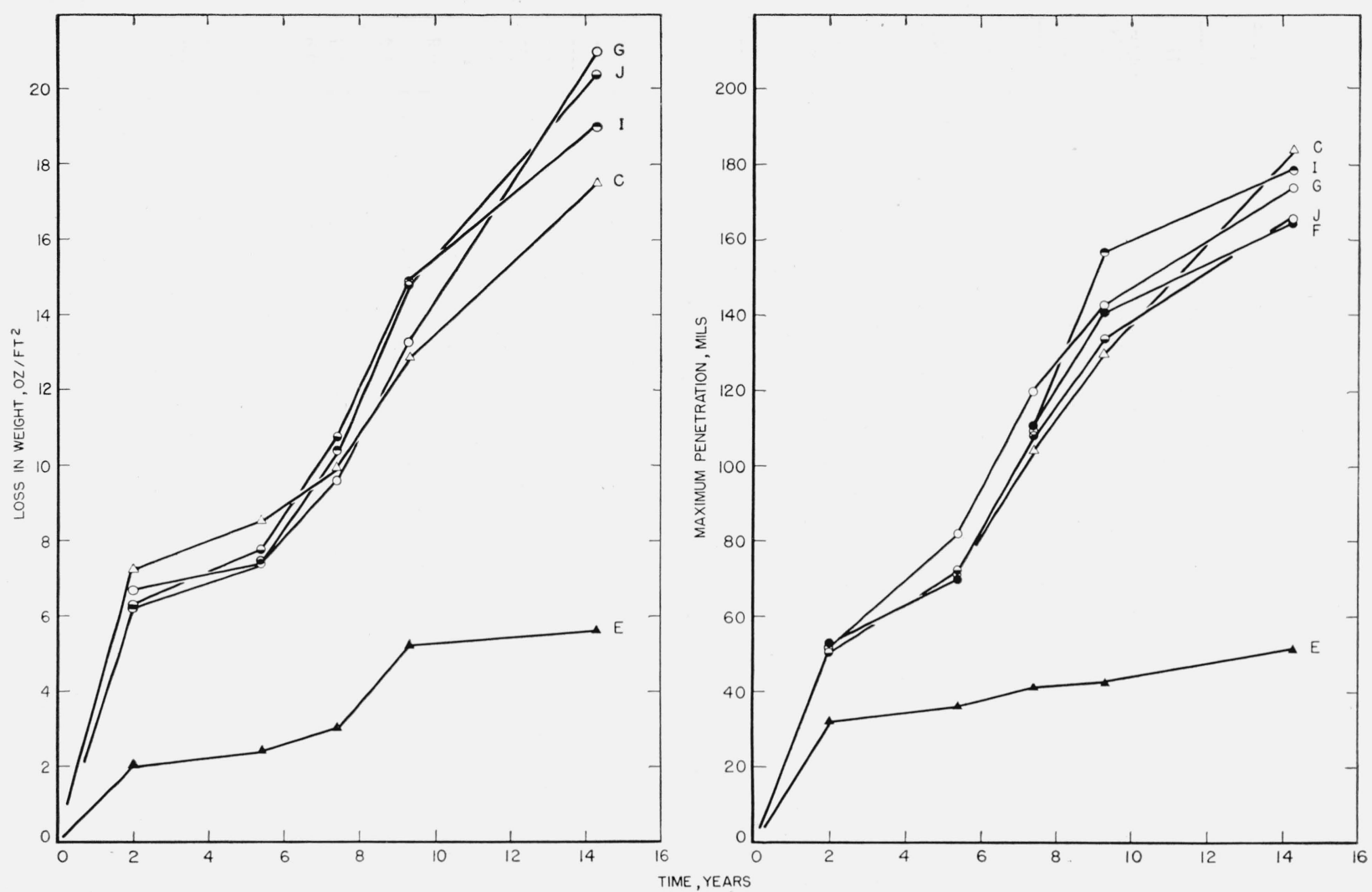

FIGURE 8. Average loss in weight and maximum penetration-time curves of cast iron pipe specimens exposed in 14 soils.

G, Rattled cast iron; F, sand-coated cast iron; I, cast iron, 0.51-precent Cu, 0.28-percent Mn; J, cast iron-0.62-percent Cu, 0.83-percent Mn: C, cast iron0.30-percent Cr, 0.15-percent $\mathrm{Ni}$; E, cast iron-15.0-percent Ni,6.6-percent $\mathrm{Cu}$. 2.6-percent $\mathrm{Cr}$

copper-molybdenum open-hearth iron $H$. Values are given for five periods of exposure except for those soils in which the number of perforations rendered the corrosion data meaningless.

It is evident from the corrosion-time curves shown in figure 13 , that the rate of corrosion with respect to both weight loss and maximum penetration varies from zero after a short period of exposure to a rate that is proportional to time.
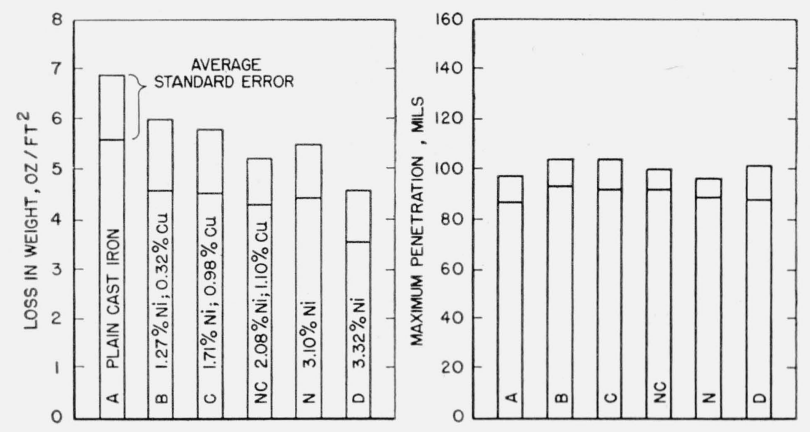

Figure 9. Average loss in weight and maximum penetration of specimens of cast irons exposed for $5 \mathrm{yr}$ in 14 soils.
In general, it may be said that curves showing a marked diminution of the rate of corrosion with time (soils 53 and 55) are typical of corrosion in well aerated soils, whereas curves indicating a linear rate (soil 56) are typical of poorly aerated soils.

Within the limits of the accuracy of the data, the curves obtained by plotting the values for maximum penetration and weight loss against the length of exposure conformed to the following equations, which were employed in comparing the corrosion of the low-alloy irons and steels:

$$
P=k T^{n},
$$

and

$$
W=k^{\prime} T^{u},
$$

where $P=$ maximum pit depth at time $T$ $W=$ weight loss at time $T$.

As will be recalled, these equations are linear on logarithmic coordinates, the constant $k\left(k^{\prime}\right)$ being the $y$-intercept and $n(u)$ the slope. The values of 
TABLE 12. Loss in weight of specimens of cast iron

(A verage of $\mathbf{2}$ specimens in ounces per square foot)

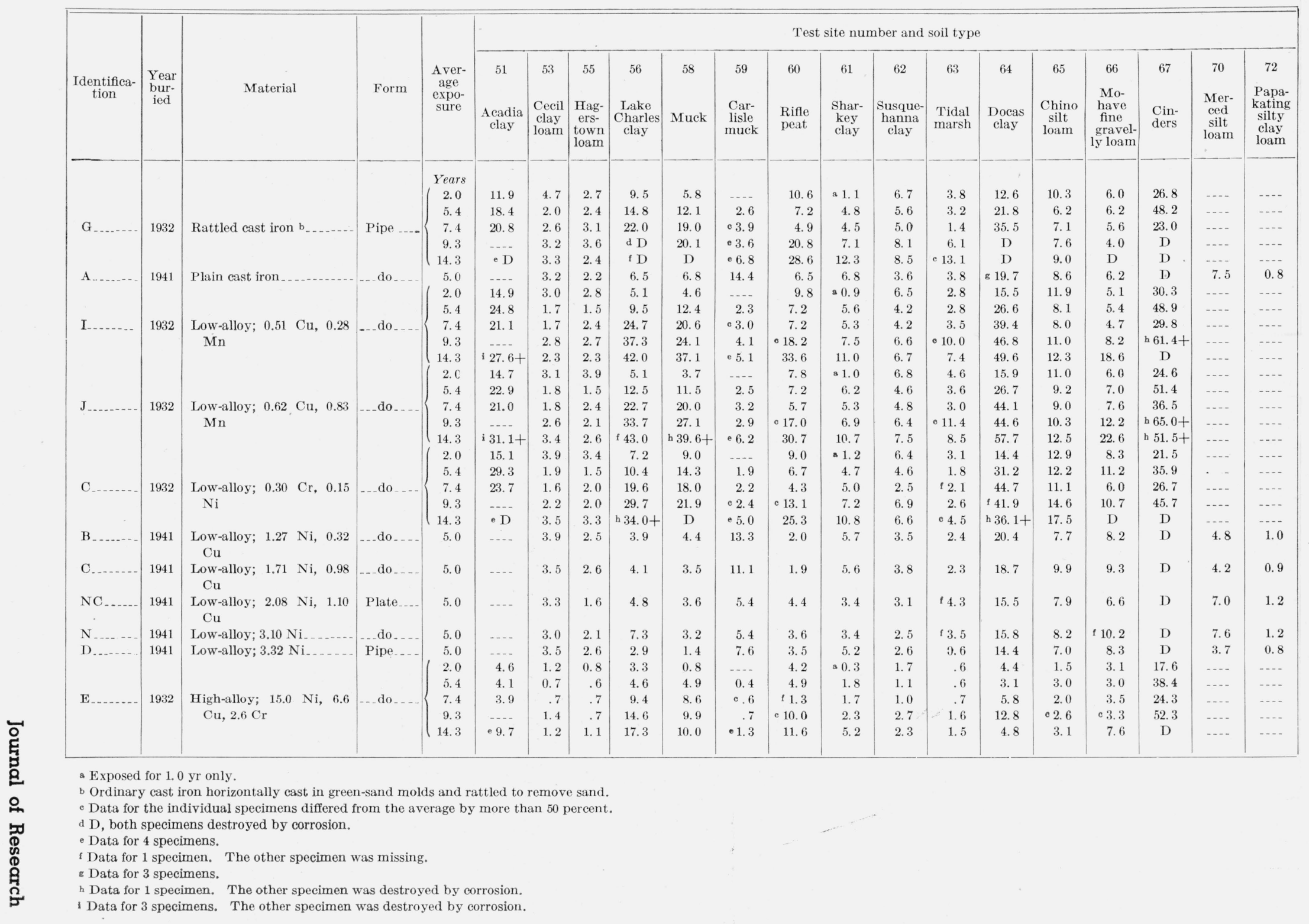




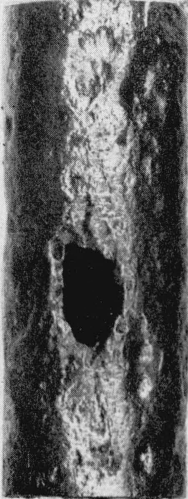

51

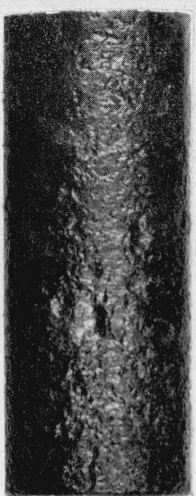

53

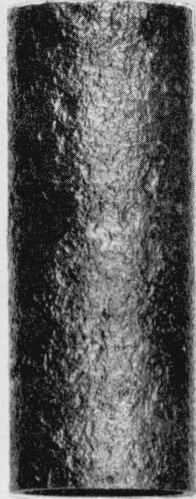

55

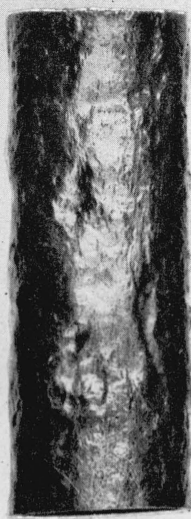

56

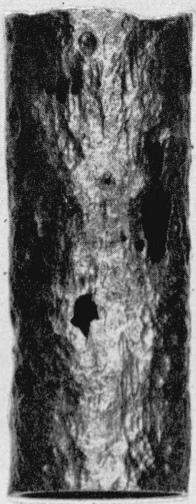

58

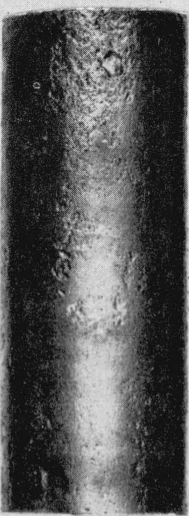

59

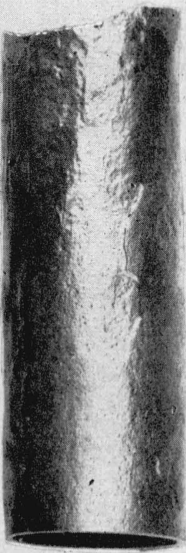

60

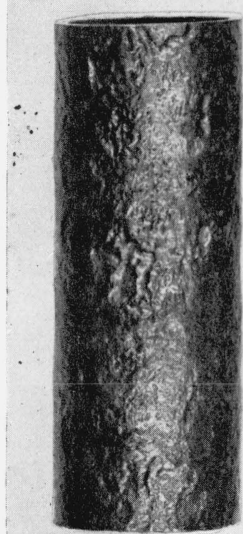

61

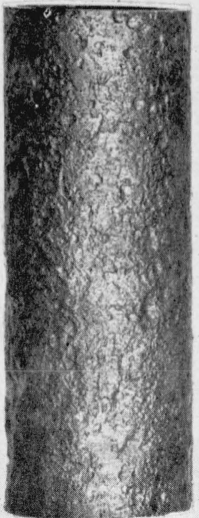

62

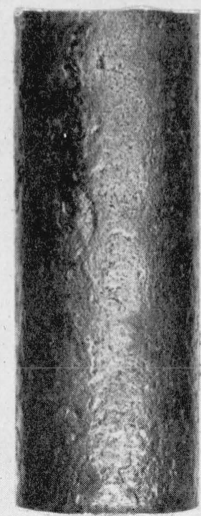

63

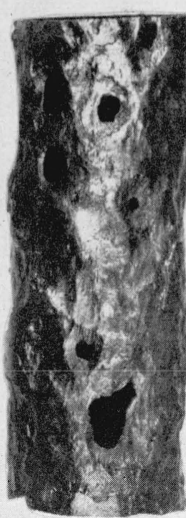

64

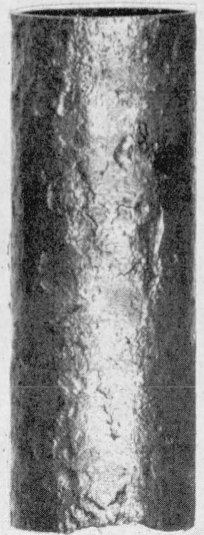

6.5

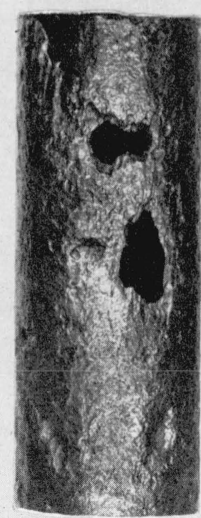

66

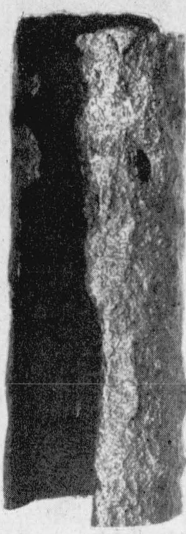

67

Figure 10. Corrosion of unalloyed steel exposed 14 yr at 14 test sites. See table 1 for identification and properties of soils. 


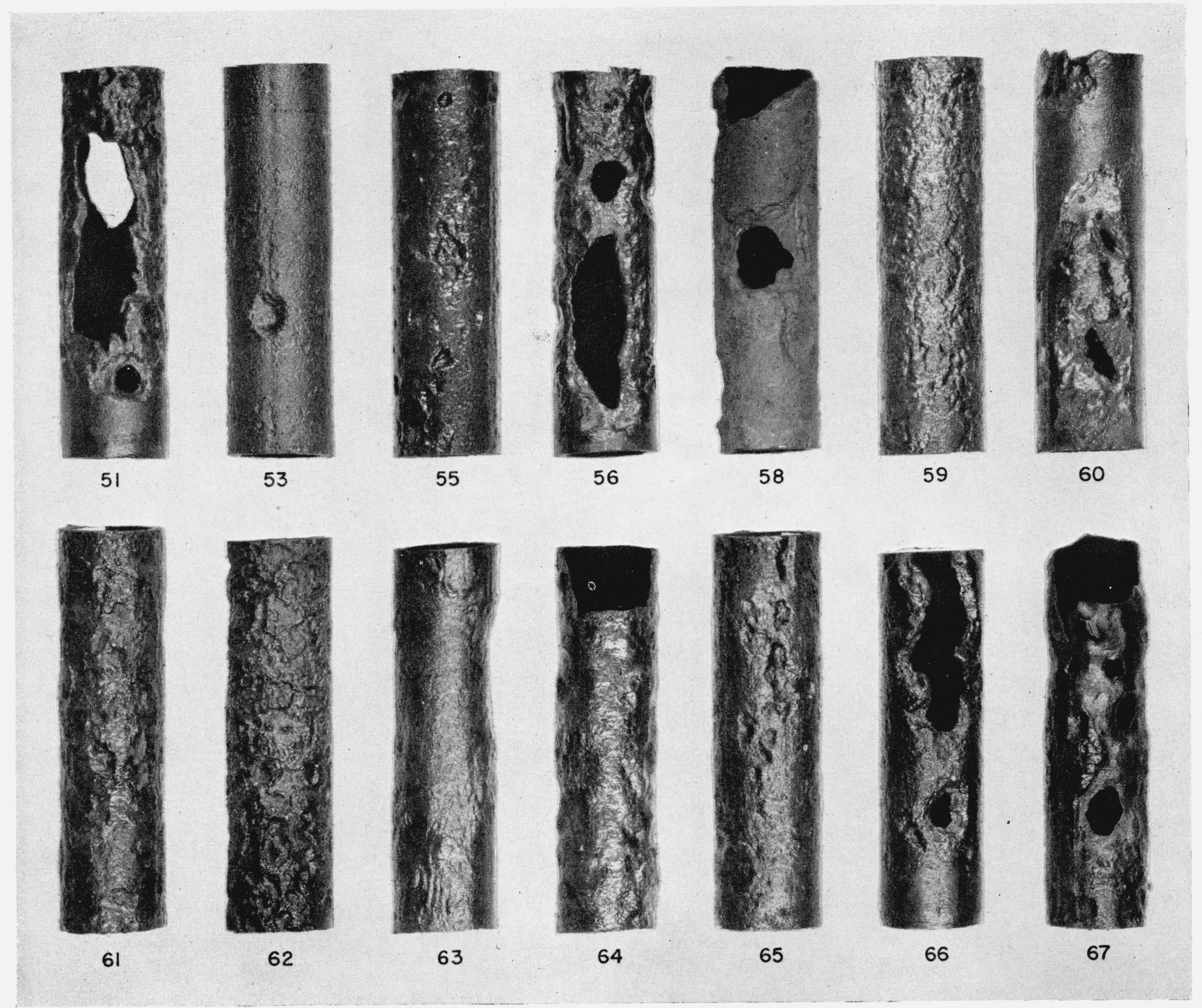

Figure 11. Corrosion of plain cast iron exposed 14 yr at 14 test sites.

See table $y$ for identification and properties of soils. 


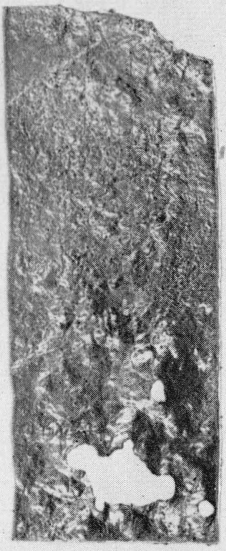

51

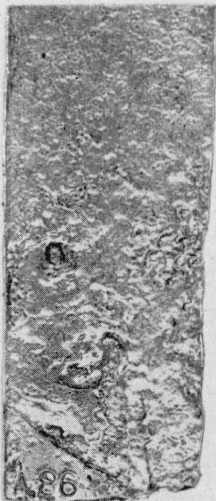

59

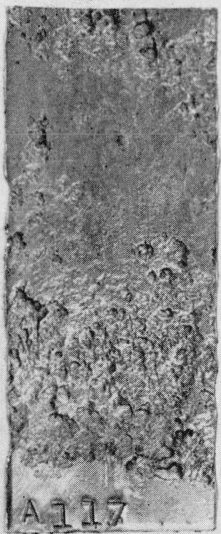

64

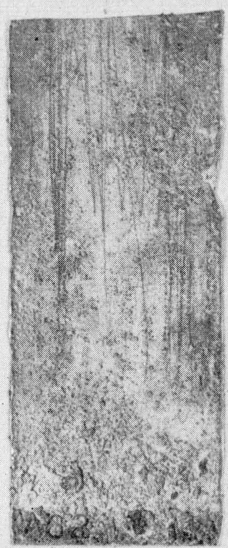

53

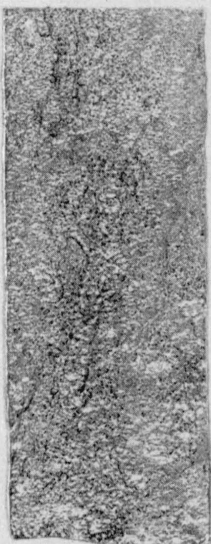

60

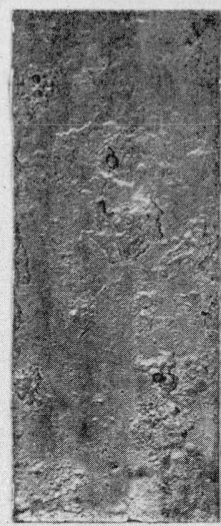

65

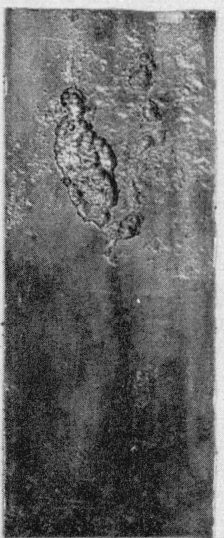

55

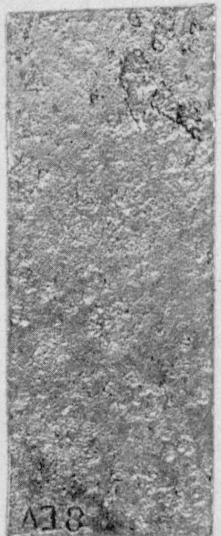

61

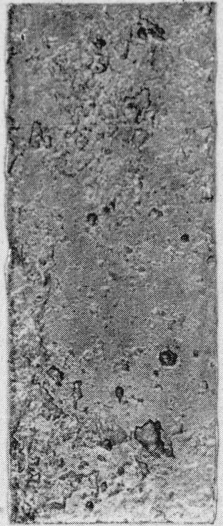

66

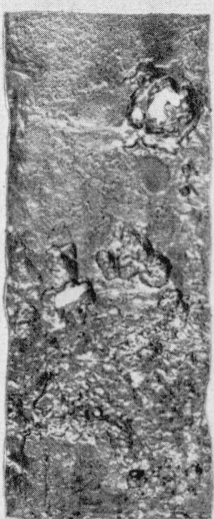

56

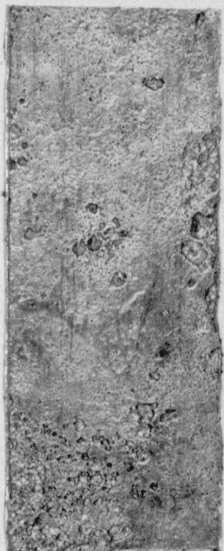

62

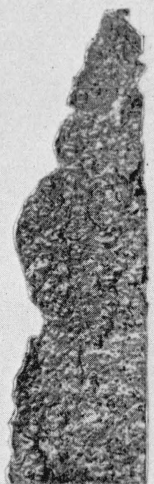

67

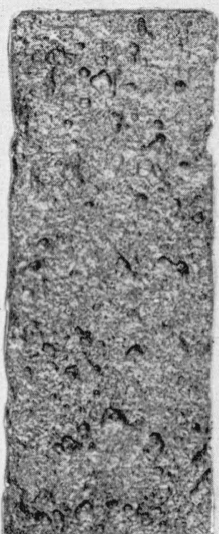

58

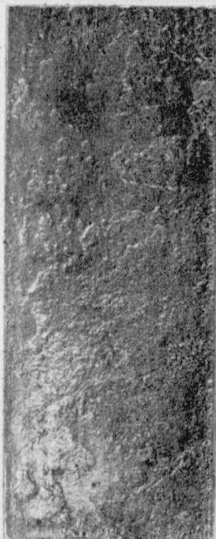

63

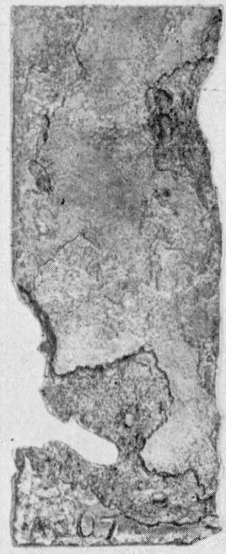

70

Figure 12. Corrosion of open-hearth iron plates exposed 9 yr at 15 test sites.

See table 1 for identification and properties of soils 

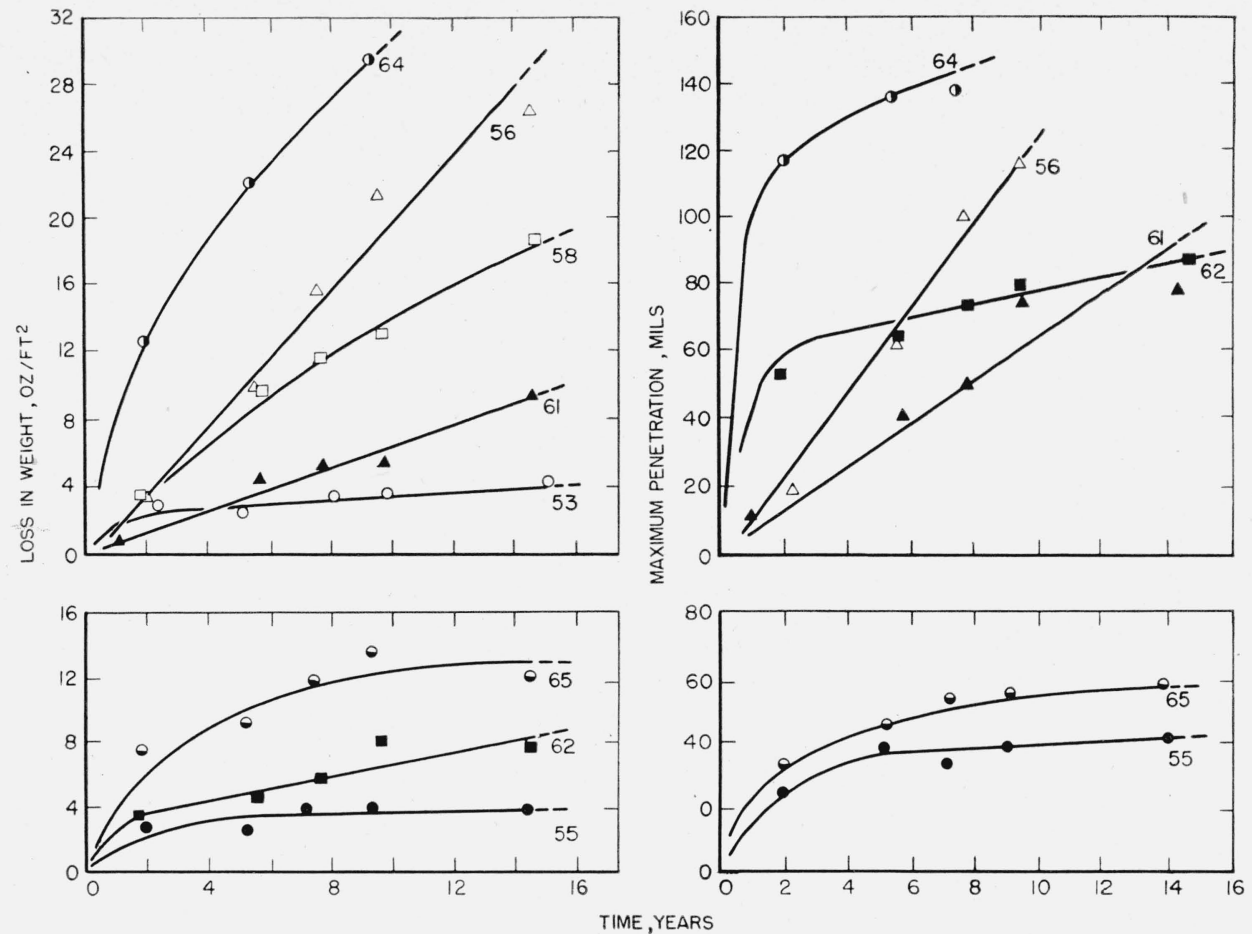

Figure 13. Weight loss and pit depth-time curves for wrought materials in typical soils

these constants, calculated according to the method of least squares, are recorded for the individual soils in table 14 . The standard errors of the constants, calculated according to the method of Ezekiel [6] are included in the table. Values for the constants $k$ and $n$ for a much larger group of soils than those under consideration at present have been reported [3].

Because the constants $k$ and $k^{\prime}$ represent, respectively, maximum penetration and weight loss at 1 yr., the values of these constants may be considered to measure approximately the initial rate of corrosion and consequently the inherent corrosiveness of the soils. The relatively large values for the constant $k$ for the group of six soils of fair to good aeration, No. 64, 66, 62, 65, 55, and 53 (table 14) are to be ascribed largely to the depolarization of the cathodic areas by the oxygen of the soil atmosphere. The low values of $k$ for the remaining poorly or very poorly aerated soils are a consequence of cathodic polarization because of the deficiency of oxygen in these soils.

Comparison of the values for $k$ and $n$ shows that there is a good inverse correlation between these two constants, from which it follows that the higher the initial rate of pitting the more rapidly does the rate of pitting decrease with time. A necessary corollary of this conclusion is that measurements of the initial rate of pitting of wrought ferrous materials in soils cannot be used for predicting corrosion over a long period unless the measurements are accompanied by some expression for the change in corrosion with time for the particular environmental conditions.

A probable explanation for the inverse correlation noted between the constants $k$ and $n$ is as follows: In poorly aerated soils, characterized by low values for the constant $k$, ferrous ions, migrating and diffusing from the local anodes have a negligible effect on the rate of corrosion, the rate being determined entirely by the depolarization of hydrogen at the cathode. However, in soils that contain oxygen in excess of that required for cathodic depolarization, ferrous ions are oxidized and precipitated in close proximity to the local anodes. The tubercles formed in this manner prevent the further migration and diffusion of ferrous ions with consequent reduction in the rate of corrosion by anodic polarization. The mechanism of tubercle formation and its significance in soil corrosion have been considered in previous papers $[8,9]$. 
A verage of 2 specimens in mils)

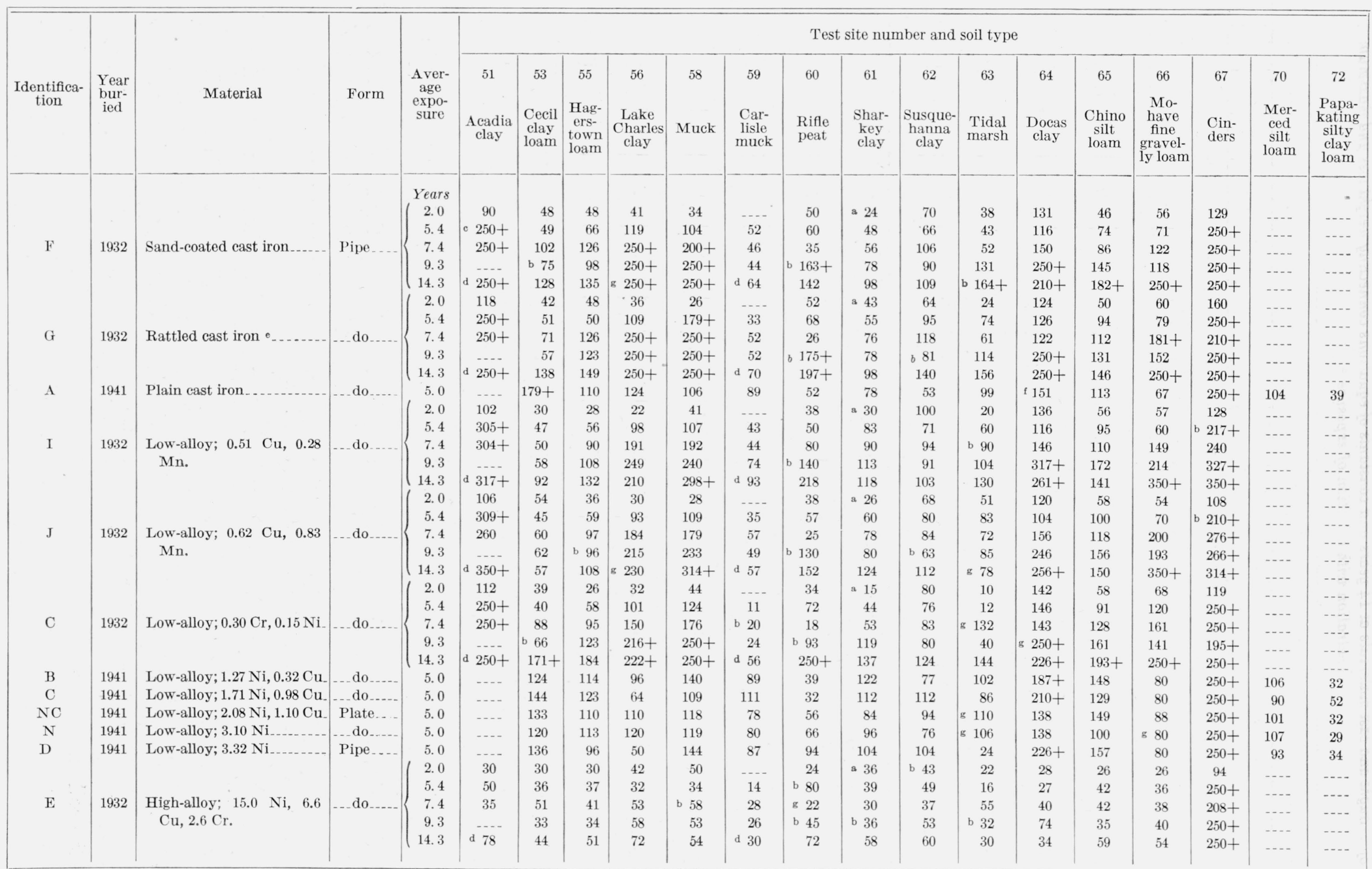

a Exposed for 1.0 yr only.

b Data for the individual specimens differed from the average by more than $50 \%$.

- + , one or more specimens contained holes because of corrosion.

d Data for 4 specimens.

- Ordinary cast iron horizontally cast in green-sand molds and rattled to remove sand. 
TABLE 14. Corrosion of wrought and cast materials at 14 years and constants of equations connecting weight loss and maximum penetration with duration of exposure a

\begin{tabular}{|c|c|c|c|c|c|c|c|c|c|c|c|c|c|c|}
\hline \multicolumn{2}{|r|}{ Soil } & \multirow{2}{*}{ Aeration } & \multicolumn{6}{|c|}{ Weight loss } & \multicolumn{6}{|c|}{ Maximum penetration } \\
\hline No. & Type & & $W_{T=14 y r}$ & $\bar{\sigma}_{P_{T=14 y r}}$ & $k^{\prime}$ & $\bar{\sigma}_{k}^{\prime}$ & $u$ & $\bar{\sigma}_{u}$ & $P_{T=14 y r}$ & $\dot{\sigma}_{P}{ }_{T=14}$ yr & $k$ & $\bar{\sigma}_{k}$ & $n$ & $\bar{\sigma}_{n}$ \\
\hline \multicolumn{15}{|c|}{ WROUGHT MATERIALS } \\
\hline & & & $o z / f t^{2}$ & $o z / f t^{2}$ & $o z / f t^{2}$ & $o z / f t^{2}$ & & & Mils & Mils & Mils & Mils & & \\
\hline 64 & $\begin{array}{l}\text { Docas clay } \\
\text { Mohave fine gravelly loam }\end{array}$ & Fair & 50.9 & 9.0 & 8.41 & 1. 71 & 0.68 & 0.13 & (b) & (n..... & $\cdots$ & $\cdots$ & $\cdots$ & $\cdots$ \\
\hline 66 & $\begin{array}{l}\text { Mohave fine gravelly loam } \\
\text { Chino silt loam }\end{array}$ & $\begin{array}{l}\text { Good } \\
\text { Gon }\end{array}$ & 15.0 & 1.6 & 7.12 & 1.12 & .28 & .09 & (b) & -.....- & $-\cdots$ & $-\cdots$ & $-\cdots$ & $-\cdots$ \\
\hline 65 & Chino silt loam & & 13.1 & 1.1 & 6.04 & 0.89 & .29 & .08 & 108 & 9 & 57.9 & 8.9 & .24 & 0.08 \\
\hline 62 & Susquehanna clay ................ & Fair & 6.9 & 1.0 & 3.18 & .87 & .29 & .14 & 83 & 5 & 55.7 & 6.4 & .15 & .06 \\
\hline 55 & Hagerstown loam & Good....... & 3.4 & 0.4 & 2. 08 & .52 & .19 & .13 & 82 & 6 & 40.8 & 5. 6 & .27 & .08 \\
\hline 53 & Cecil clay loam & $\ldots$......... & 4.2 & .4 & 2. 46 & .44 & .20 & .09 & 77 & 8 & 34.1 & 6.3 & .31 & .09 \\
\hline 60 & Rifle peat $\ldots \ldots \ldots$ & Poor ......... & 17.8 & 6.2 & 2. 61 & 1. 78 & .73 & .33 & 56 & 16 & 16.4 & 9.0 & .46 & .27 \\
\hline 63 & Tidal marsh & Very poor_.. & 7.7 & 1.5 & 1. 58 & 0.64 & .60 & .20 & 81 & 11 & 13.8 & 3.8 & .67 & .14 \\
\hline 61 & Sharkey clay & Poor ......... & 9.3 & 0.8 & 1. 12 & .14 & .80 & .07 & 92 & 8 & 13.5 & 1. 7 & .72 & .06 \\
\hline 56 & Lake Charles clay ........ & Very poor... & 30.5 & 2.3 & 1. 72 & .25 & 1.09 & .08 & (b) & -..... & $\ldots$ & $\ldots$ & $\ldots$ & $\ldots$ \\
\hline 58 & Muck . . . & Poor & 21.0 & 1.8 & 1.85 & .31 & 0.92 & .08 & 194 & 54 & 9.1 & 2.8 & 1.16 & .17 \\
\hline 59 & Carlisle muck . . & Very poor... & 4.1 & 0.1 & 0.49 & .02 & .81 & .02 & 36 & 2 & 4.4 & 0.7 & 0.80 & .07 \\
\hline 51 & Acadia clay & Poor ......... & 20.9 & .2 & 5. 59 & 10 & .50 & .09 & (b) & (...... & $-\ldots$ & -... & $-\ldots$ & -...- \\
\hline 67 & Cinders & Very poor..- & 41.0 & 3.0 & 8.67 & .76 & .59 & .06 & (b) & -..... & -... & -... & -... & .... \\
\hline \multicolumn{15}{|c|}{ CAST MATERIALS } \\
\hline 64 & Docas clay & Fair .... & 58.0 & 5.8 & 9.12 & 1.4 & 0.70 & 0.09 & 140 & 20 & 120 & 20 & 0.06 & 0.10 \\
\hline 62 & Susquehanna clay .............. & $\ldots$ do & 5.9 & 1.1 & 5.53 & 1.8 & .03 & .16 & 98 & 10 & 70.5 & 14.0 & .13 & .10 \\
\hline 65 & Chino silt loam & Good & 10.7 & 1. 4 & 9.91 & 2.4 & .03 & .12 & 163 & 14 & 40.5 & 6.4 & .53 & .08 \\
\hline 66 & Mohave fine gravelly loam & Fair.......... & 8.0 & 1.5 & 5. 78 & 0.68 & .12 & .16 & 224 & 58 & 34.5 & 13.3 & .71 & .22 \\
\hline 53 & Cecil clay loam & Good & 2.4 & 0.5 & 3.13 & 1.4 & -.11 & .22 & 81 & 10 & 28.5 & 6.8 & .39 & .12 \\
\hline 61 & Sharkey clay & Poor ........ & 10.1 & 1.0 & 1. 12 & 0.17 & .84 & .08 & 110 & 8 & 28.1 & 3.6 & .52 & .05 \\
\hline 60 & Rifle peat & $\ldots$ do $\ldots \ldots$ & 17.0 & 8.0 & 4.45 & 4.3 & .51 & .43 & 72 & 68 & 23.7 & 29.0 & .42 & .59 \\
\hline 55 & Hagerstown loam & Good & 2.3 & 0.4 & 2.82 & 0.96 & -.07 & .17 & 146 & 17 & 20.5 & 4.3 & .74 & .11 \\
\hline 58 & Muck . . . & Poor ........ & 32.0 & 2.4 & 3.05 & .35 & .89 & .06 & 369 & 26 & 14.9 & 1.3 & 1. 22 & .05 \\
\hline 63 & Tidal marsh & Very poor... & 6.1 & 2.3 & 1. 96 & 1.5 & .70 & .09 & 126 & 7 & 14.8 & 1.8 & 0.81 & .06 \\
\hline 56 & Lake Charles clay ................ & $\ldots$ do $\ldots \ldots . . .$. & 40.5 & 6.8 & 3.12 & 1.0 & .97 & .16 & 369 & 51 & 12.1 & 2.0 & 1. 30 & .11 \\
\hline 59 & Carlisle muck . . . . . . . . & $\ldots$ do $\ldots . . . .$. & 5.4 & 0.5 & 0.51 & 0.16 & .89 & .14 & 67 & 11 & 10.5 & 4.3 & 0.70 & .26 \\
\hline 51 & A cadia clay & Poor ......... & 29.6 & 6.0 & 11.4 & .96 & .36 & .16 & (b) & -..... & $-\cdots$ & $-\cdots$ & -... & $-\cdots$ \\
\hline 67 & Cinders & Very poor... & 60.3 & 14.0 & 19.4 & 7.0 & .43 & .20 & (b) & - n & -..- & $\ldots$ & $-\cdots$ & $\cdots$ \\
\hline
\end{tabular}

- $W=k^{\prime} T^{u}, P=k T^{n}$, where $W=$ weight loss at the time, $T$; and $P=\operatorname{depth}$ of the deepest pit at the time, $T$.

b Specimens perforated at an early period. See table 6 for data on pit depths.

Although the permeability of the soil to air appears to be the major factor in determining the value of the constant $n$, it should be noted that any property of the soil that tends to increase the solubility of ferrous ions at the local anodes, such as high contents of chloride, sulfate, or hydrogen ions, would also have the effect of increasing the magnitude of $n$ and consequently the depths of the deepest pits.

The inverse correlation between the values of the constants for maximum penetration, $k$ and $n$, is also to be noted between the constants for weight loss, $k^{\prime}$ and $u$, showing that weight loss and pitting follow the same general tendencies with respect to the soil environment.

The average values of the constants $k, k^{\prime}, n$, and $u$ for the cast materials that corroded at approximately the same rates are also presented in table 14. The materials represented are plain cast iron $G$, and the low-alloy cast irons $C, I$, and $J$. In this table the same general tendencies noted in the case of the wrought materials are to be observed, but with more numerous exceptions.

\section{Comparison of Wrought and Cast Materials}

Because of dissimilarities in the dimensions of the wrought and cast ferrous specimens in the previous field tests, no comparison could be made of the relative corrodibility of the two classes of materials. However, in the present series of tests, the dimensions of the cast and wrought specimens were similar, so that comparisons between the two materials are possible. 
The cast and wrought materials were compared by the method previously described in connection with the comparison with plain steel of the low-alloy wrought materials exposed $14 \mathrm{yr}$. The differences between the values of weight loss and pitting for cast iron and steel at $14 \mathrm{yr}$ recorded in table 14 for the different soils were calculated, and the standard errors of these differences were computed by means of eq 6 . These data are given in table 15 .

Because the differences between the mean values for weight loss of the cast and wrought materials were, with few exceptions, less than twice the respective standard errors of the differences, it is necessary to conclude that no generally significant difference between the weight losses of the two materials can be detected. With respect to maximum penetration, differences less than twice the standard error of the difference were noted in four of the nine soils for which the data could be statistically treated. In the remaining five soils, the greater depth of pits on the cast specimens should be considered significant.

\section{Summary}

This report contains the results of measurements of corrosion made on a variety of wrought and cast ferrous materials after exposure to different soil conditions for periods up to $14 \mathrm{yr}$. Steels containing small amounts of nickel and chromium showed increased resistance to soil corrosion, but the resulting improvement was small. However, certain wholly austenitic steels containing high percentages of chromium and nickel were completely resistant to corrosion. In those soils in which there was a significant difference in the pitting of cast iron and wrought materials, cast iron usually pitted at somewhat higher rates. High sustained rates of corrosion occurred generally in poorly aerated soils high in soluble salts or in acidity. In well aerated soils low in soluble salts, corrosion virtually ceased after a relatively short period because of the formation of layers of corrosion products close to the metal surface.

TABLE 15. Comparison of wrought and cast materials at 14 years

\begin{tabular}{|c|c|c|c|c|c|c|c|c|c|c|c|c|c|c|}
\hline \multirow{4}{*}{ Soil a } & \multicolumn{7}{|c|}{ Loss in weight $\left(\mathrm{oz} / \mathrm{ft}^{2}\right)$} & \multicolumn{7}{|c|}{ Maximum pit depth (mils) } \\
\hline & \multicolumn{2}{|c|}{$\begin{array}{l}\text { Wrought } \\
\text { materials }\end{array}$} & \multicolumn{2}{|c|}{ Cast materials } & \multirow{3}{*}{$\begin{array}{c}\text { Differ- } \\
\text { ence } \\
\text { between } \\
\text { the } \\
\text { means } \\
\bar{X}_{1}-\bar{X}_{2}\end{array}$} & \multirow{3}{*}{$\begin{array}{c}\text { Standard } \\
\text { error } \\
\text { of the } \\
\text { differ- } \\
\text { ence b } \\
\bar{\sigma}_{D}\end{array}$} & \multirow[b]{3}{*}{$2 \sigma_{D}$} & \multicolumn{2}{|c|}{$\begin{array}{l}\text { Wrought } \\
\text { materials }\end{array}$} & \multicolumn{2}{|c|}{ Cast materials } & \multirow{3}{*}{$\begin{array}{c}\text { Differ- } \\
\text { ence } \\
\text { between } \\
\text { the } \\
\text { means } \\
\bar{X}_{1}-\bar{X}_{2}\end{array}$} & \multirow{3}{*}{$\begin{array}{c}\text { Standard } \\
\text { error } \\
\text { of the } \\
\text { differ- } \\
\text { ence b } \\
\bar{\sigma}_{D}\end{array}$} & \multirow[b]{3}{*}{$2 \sigma_{D}$} \\
\hline & Mean & $\begin{array}{l}\text { Stand- } \\
\text { ard } \\
\text { error }\end{array}$ & Mean & $\begin{array}{l}\text { Stand- } \\
\text { ard } \\
\text { error }\end{array}$ & & & & Mean & $\begin{array}{l}\text { Stand- } \\
\text { ard } \\
\text { error }\end{array}$ & Mean & $\begin{array}{l}\text { Stand- } \\
\text { ard } \\
\text { error }\end{array}$ & & & \\
\hline & $\bar{X}_{1}$ & $\bar{\sigma}_{1}$ & $\bar{X}_{2}$ & $\overline{\boldsymbol{\sigma}}_{2}$ & & & & $X_{1}$ & $\bar{\sigma}_{1}$ & $\bar{X}_{2}$ & $\bar{\sigma}_{2}$ & & & \\
\hline 51 & 20.9 & 0.2 & 29.6 & 6.0 & -8.7 & 6.0 & 12.0 & $\ldots$ & .... & $\ldots$ & .... & $\ldots$ & ..... & ..... \\
\hline 53 & 4. 2 & .4 & 2.4 & 0.5 & +1.8 & 0.7 & 1.4 & 77 & 8 & 81 & 10 & -4 & 12 & 24 \\
\hline 55 & 3.4 & .4 & 2.3 & $: 4$ & +1.1 & .6 & 1.2 & 82 & 6 & 146 & 17 & -64 & 18 & 36 \\
\hline 56 & 30.5 & 2.3 & 40.5 & 6.8 & -10.0 & 7.2 & 14.4 & -.... & .... & .... & -... & ..... & -..... & -... \\
\hline 58 & 21.0 & 1.8 & 32.0 & 2.4 & -9.0 & 3.0 & 6. 0 & 194 & 54 & 369 & 26 & -175 & 60 & 120 \\
\hline 59 & 4.1 & 0.1 & 5.4 & 0.5 & -1.2 & 0.5 & 1.0 & 36 & 2 & 67 & 11 & -30 & 11 & 22 \\
\hline 60 & 17.8 & 6.2 & 17.0 & 8.0 & +0.8 & 10.1 & 20.2 & 56 & 16 & 72 & 68 & -16 & 70 & 140 \\
\hline 61 & 9.3 & 0.8 & 10.1 & 1.0 & -.8 & 1.3 & 2. 6 & 92 & 8 & 110 & 8 & -18 & 11 & 22 \\
\hline 62 & 6.9 & 1.0 & 5.9 & 1.1 & +.9 & 1.5 & 3.0 & 83 & 5 & 98 & 10 & -16 & 11 & 22 \\
\hline 63 & 7.7 & 1.5 & 6.1 & 2.3 & +1.6 & 2.8 & 5. 6 & 81 & 11 & 126 & 7 & -45 & 13 & 26 \\
\hline 64 & 50.9 & 9.0 & 58.0 & 5.8 & -7.1 & 10.7 & 21.4 & ..... & .... & ..... & ...- & ..... & ....... & ..... \\
\hline 65 & 13.1 & 1.1 & 10.7 & 1.4 & +2.4 & 1.8 & 3.6 & 108 & 9 & 163 & 14 & -55 & 17 & 33 \\
\hline 66 & 15.0 & 1.6 & 8. 0 & 1.5 & +7.0 & 2.2 & 4.4 & ..... & -... & -.... & -... & -...- & -.... & -.... \\
\hline 67 & 41.0 & 3.0 & 60.3 & 14.0 & -19.0 & 14.3 & 28.6 & - . . & -... & -.... & .... & .... & .... & $\ldots$ \\
\hline
\end{tabular}

s See table 1 for type, location, and properties of soils,

$\sigma_{D}=\sqrt{\frac{\sigma_{1}^{2}}{N_{1}}+\frac{\sigma_{2}^{2}}{N_{2}}}$ (see text) 
The field tests described in this paper were planned and installed, and until 1946 were conducted under the supervision of K. H. Logan. The measurements of corrosion were made chiefly by Warren P. Dettmers, who assisted in other capacities.

\section{References}

[1] Kirk H. Logan, Underground corrosion, NBS Circular C450 (1945).

[2] Melvin Romanoff, J. Research NBS 34, 227 (1945) RP1639.

[3] K. H. Logan, S. P. Ewing, and I. A. Denison, Symposium on corrosion testing procedures, Am. Soc. Test. Mats. (Philadelphia, Pa., 1937).
[4] L. M. Martin, NBS Soil Corrosion Conference 1937, Unpublished.

[5] Mordecai Ezekiel, Methods of correlation analysis (John Wiley and Sons, Inc., New York, N. Y., 1930).

[6] Herbert Arkin and Raymond R. Colton, Statistical methods (Barnes and Noble, New York, N. Y., 1935).

[7] Herbert J. French and Francis L. LaQue, Alloy constructional steels, Am. Soc. Metals (Cleveland, Ohio, 1942).

[8] I. A. Denison and R. B. Darnielle, Trans. Electrochem. Soc. $\boldsymbol{\gamma 6}, 199$ (1939).

[9] I. A. Denison, Chemical aspects of underground corrosion and corrosion prevention, Monograph Am. Gas Assoc. (1948).

Washington, June 10, 1949. 